\title{
40th Biennial American Cytogenetics Conference
}

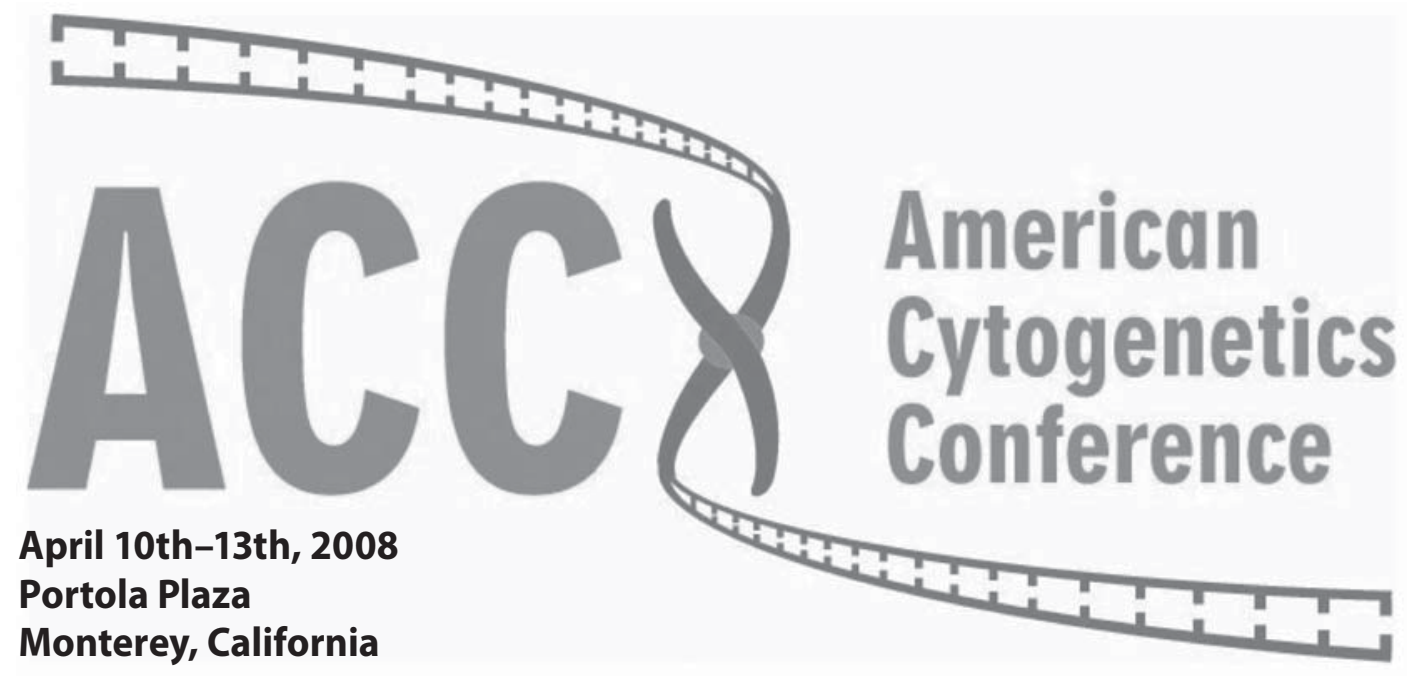

Meeting Co-Chairs:

Lauren Jenkins (Facilities)

Susan Zneimer (Program)

Recipient of Student Travel Awards:

Sponsored by Genetix/Applied Imaging Corporation and Genzyme Genetics

Kimberley Haydu - Virginia Commonwealth University, Richmond, VA

Feng Li - McKusick-Nathans Institute of Genetic Medicine, Baltimore, MD

Paulie Papavassiliou - Virginia Commonwealth University, Richmond, VA

American Cytogenetics Conference Distinguished Cytogeneticist Award

Dr. Orlando J. Miller and Dr. Dorothy Anne Miller

The 2008 American Cytogenetics Conference gratefully acknowledges the generous support of the following companies:

Affymetrix

Alphelys

BCM Medical Genetics Laboratories

Blue Gnome Ltd

Cytocell/Rainbow Scientific, Inc.

Genetix/Applied Imaging Corporation

Genomic Software

Ikonisys

Invitrogen

LabCorp

Oxford Gene Technology

Quest Diagnostics Nichols Institute

SciGene

Tecan US, Inc.
Agilent Technologies

Applied Spectral Imaging, Inc.

BioDiscovery, Inc.

Combimatrix Molecular Diagnostics

GeneDx

Genial Genetic Solutions Ltd/Rainbow Scientific, Inc

Genzyme Genetics

InfoQuant Ltd

Karger

Metasystems

Perkin Elmer

SCC Soft Computer

Signature Genomic Laboratories 


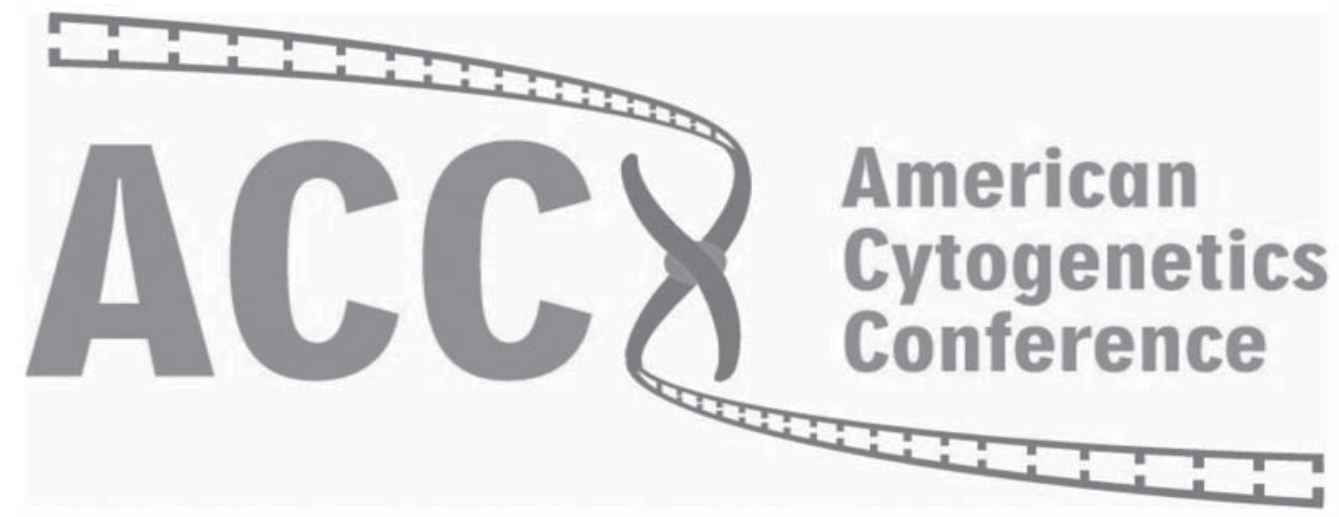

\section{Meeting Organizers and ACC Officers}

Program Committee

Susan Zneimer

Athena Cherry

Hutton Kearney

Urvashi Surti

Denise Quigley

Daynna Wolff

Distinguished Cytogeneticist

Art Brothman

Committee

Susan Olson

Harold Klinger Memorial Award

Peter Jacky

Jim Mascarello

Student Awards

Eileen Bryant

Gopalrao Velagaleti

Corporate Sponsorships

Barbara Dupont

Gail Wenger

Membership

Colleen Jackson-Cook

Kristin May

Publicity

Frank Grass

Wendy Golden

Registration

Sue Ann Berend

Joey Kelly

Website

Robert Best

Historian

Charleen Moore

Continuing Education

Athena Cherry

2006-2008 ACC Officers and Board of Directors

President: Lauren Jenkins

Vice President: Avirachan Tharapel

Secretary/Treasurer: Sue Ann Berend

Past President: Debra Saxe

\section{Board of Directors:}

Art Brothman

Bob Best

Susan Olson 


\section{Exhibitors/Sponsors}

Thanks to all the exhibitors for supporting this meeting. Please visit their booths during the breaks!

Affymetrix, Inc.

3420 Central Expressway

Santa Clara, CA 95051

Phone: (888) 362-2447

Fax: (408) 731-5447

Contact: Karen Morrison

Karen_morrison@affymetrix.com

Alphelys

526 S. Main St., Ste 714G

Akron, $\mathrm{OH} 44311$

Phone: (866) 840-3758

Fax: (330) 252-2713

Contact: Luigi Armogida

luigi@nichevision.com

BCM Medical Genetics Laboratories

Baylor College of Medicine

One Baylor Plaza, NAB 2015

Houston, TX 77030

Phone: (713) 798-8389

Fax: (713) 798-4187

Contact: Michael Frazier

mfrazier@bcm.tmc.edu

Blue Gnome Ltd

Breaks House

Great Shelford, Cambridge

CB22 5LD, UK

Phone: +44 (0)1223 844441

Fax: +44 (0)1223 844445

Contact: David Chrimes, $\mathrm{PhD}$

David.chrimes@cambridgebluegnome.com

Cytocell/Rainbow Scientific, Inc.

83 Maple Avenue

Windsor, CT 06095

Phone: (860) 298-8382

Fax: (860) 298-8586

Contact: Peter Mousseau

info@rainbowscientific.com

Genetix/ Applied Imaging Corp.

120 Baytech Dr.

San Jose, CA 95134

Phone: (800) 634-6322

Fax: (408) 719-6401

Contact: Chris Cruze

ccruze@aicorp.com
Agilent Technologies, Inc.

5301 Stevens Creek Boulevard

MS 53UWG

Santa Clara, CA 95051-7201

Phone: (408) 553-6217

Fax:(408) 553-7100

Contact: Condie Carmack, PhD

zondie_carmack@agilent.com

Applied Spectral Imaging, Inc.

1497 Poinsettia Ave, \#158

Vista, CA 92081

Phone: (760) 929-2840

Fax: (760) 929-2842

Contact: F. Scott Cowan Jr

Scott.cowan@spectral-imaging.com

BioDiscovery, Inc.

2121 Rosecrans Ave., Suite 3315

El Segundo, CA 90245 USA

Phone: (310)-414-8100

Fax: (310)-414-8111

Contact: Crystal Jones

cjones@biodiscovery.com

Combimatrix Molecular Diagnostics

310 Goddard, Ste 150

Irvine, CA 92618

Phone: (800) 710-0624

Fax: (949) 753-1504

Contact: Dindy Ramkissoon

Aleli Hernandez

dindy@cmdiagnostics.com

ahernandez@cmdiagnostics.com

GeneDx

207 Perry Parkway, Ste 6

Gaithersburg, MD 20877

Phone: (301) 519-2100

Fax: (301) 519-2892

Contact: Swaroop Aradhya, PhD

swaroop@genedx.com

Genial Genetics Solutions Ltd./

Rainbow Scientific, Inc.

83 Maple Avenue

Windsor, CT 06095

Phone: (860) 298-8382

Fax: (860) 298-8586

Contact: Peter Mousseau

info@rainbowscientific.com 
Genomic Software, Inc.

1000 Escalon Ave.

Sunnyvale, CA 94085

Phone: (408) 761-6227

Fax: (408) 395-5010

Contact: John Mann, MD

johnmannmd@genomicsoftware.com

Ikonisys

5 Science Park

New Haven, CT 06511

Phone: (203) 776-0791

Fax: (203) 776-0795

Contact: Rich DeLaby

Rich.delaby@ikonisys.com

Invitrogen Corporation

3175 Staley Road

Grand Island, NY 14072

Phone: (800) 955-6288 X46993

Fax: (716) 774-6996

Contact: Karissa Morcelle

Karissa.morcelle@invitrogen.com

Lab Corp

500 South Main St, Ste 301

Burlington, NC 27715

Phone: (919) 361-7234

Fax: (919) 361-7149

Contact: Annette Martin

Martina@labcorp.com

Oxford Gene Technology

Begbroke Science Park

Sandy Lane, Yarnton

Oxford OX5 1PF UK

Phone: +44 (0)1865 856826

Fax: +44 (0)1865 848684

Contact: Nicola Booton-Mander

Nicola.booton-mander@ogt.co.uk

Quest Diagnostics Nichols Institute

14225 Newbrook Drive

Chantilly, VA 20151

Phone: (703) 802-7094

Fax: (703) 802-7103

Contact: Philip N. Mowrey, PhD, Joey Kelly, PhD

philip.n.mowrey@questdiagnostics.com

Joey.c.kelly@questdiagnostics.com

SciGene

306 Potrero Avenue

Sunnyvale, CA 94085

Phone: (800) 342-2119

Fax: (408) 733-7336

Contact: Tony Lialin

tlialin@scigene.com

Tecan US, Inc.

5022 Stirrup Creeek, Suite 310

Durham, NC 27709

Phone: (617) 395-4195

Fax: (919) 361-5201

Contact: Camilo Canel

Camilo.Canel@Tecan.com
Genzyme Genetics

3400 Computer Drive

Westborough, MA 01581

Phone: (360) 601-2434

Fax: (508) 286-2505

Contact: Kirk Erickson

Kirk.Erickson@genzyme.com

InfoQuant Ltd

35 Seacon Tower

London, UK E14 8JX

Phone: (617) 475-5167

Contact: Anton Petrov

anton@infoquant.com

Karger

P.O Box

CH-4009 Basel (Switzerland)

phone: +416130611 11

fax: +4161306 1234

Contact: Stefan Goldbach

s.goldbach@karger.ch

MetaSystems Group, Inc.

24 Church St.

Watertown, MA 02472

Phone: (617) 924-9950

Fax: (617) 924-9954

Contact: Ulrich Klingbeil, Bill Hanifin

Heather Whitney

uk@metasystems

bhanifin@metasystems.com

hwhitney@metasystems.org

PerkinElmer

710 Winter Street

Waltham, MA 02451

Phone: (800) 762-4000

Fax: (203) 944-4904

Contact: Brandon Perthuis

Brandon.Perthuis@perkinelmer.com

SCC Soft Computer

5400 Tech Data Drive

Clearwater, FL 33760

Phone: (727) 789-0100

Fax: (727) 789-0124

Contact: Ellie Vahman

ellie@softcomputer.com

Signature Genomic Laboratories 120 North Pine Street, Suite 242C

Spokane, WA 99202

Phone: (877) 744-2447

Fax: (509) 474-6839

Contact: Lisa G. Shaffer, PhD

shaffer@signaturegenomics.com

Preprint Cytogenet Genome Res 121/1/2008

Abstracts will be available online,

free of charge 


\section{Drs. O.J. and D.A. Miller: 2008 Co-recipients, ACC Distinguished Cytogeneticists}

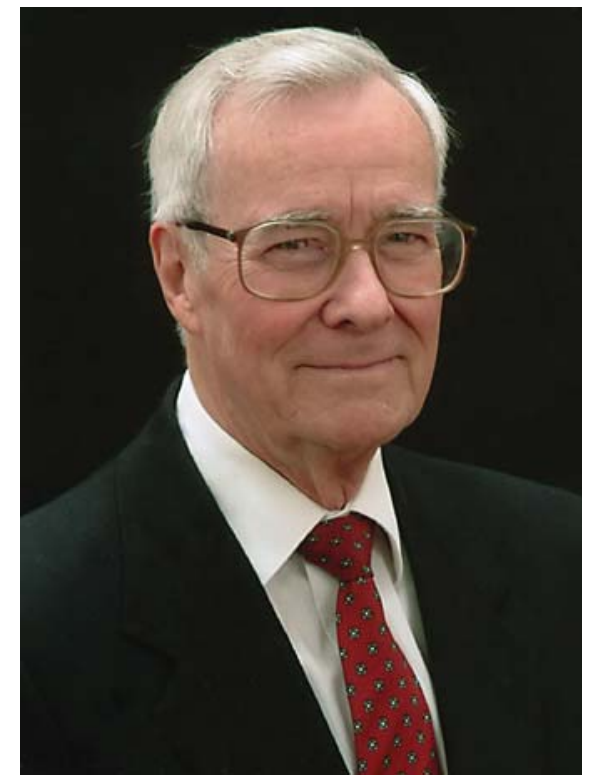

Orlando Jack Miller

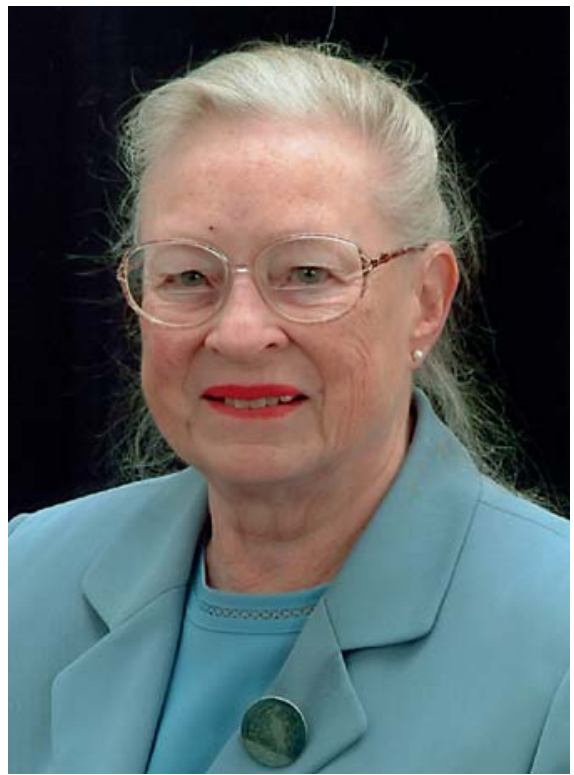

Dorothy Anne Miller
Orlando (Jack) Miller, M.D. (1950, Yale) and Dorothy Anne (Sandy) Miller, Ph.D. (1957, Yale), are the co-recipients of the ACC's Distinguished Cytogeneticist Award for 2008. While they have made significant independent contributions, their joint research has had a larger impact on human and mammalian cytogenetics. They have trained more than 60 graduate students and fellows, served on numerous editorial and scientific review boards, and published over 250 papers, aided by grants to one or the other as Principal Investigator from the NIH, NSF, March of Dimes, and other organizations. They have influenced two generations of geneticists during their more than 40 years of research, teaching and service.

Jack, an obstetrician and gynecologist, received training in genetics (1958-1960) with Professor Lionel S. Penrose at the Galton Laboratory, University College London. They helped usher in the field of human cytogenetics by reporting, with Charles Ford, the first XXY/21-trisomic male (1959) and, independently, an XXYY male (1961). Jack moved to Columbia University in 1960 and, with Roy Breg at Yale, found still more chromosomal causes of infertility and mental retardation, e.g., an XXXXY male (1961). Jack spent a sabbatical year, 19681969, with Professor Henry Harris at Oxford University. Using the new technique of Sendai virus-induced somatic cell hybridization, they produced the first evidence for the existence of tumor suppressor genes.

In 1964, when their children were 4, 6, and 8 years old, Jack's wife Sandy joined his research group and played an increasingly important role, especially in chromosome banding, mapping and comparative cytogenetics. The O.J./D.A. Miller team were the first to use interspecific somatic cell hybrids with a chromosome banding technique to assign a gene to a specific autosome (1971). They were the first to identify every mouse chromosome by a banding technique, to assign most of the mouse linkage groups to specific chromosomes, and to develop a simple way to determine the centromeric end of mouse linkage groups (1971). With Dr. B.F. Erlanger, they produced Q-, G-, R- and other banding patterns using only antibodies to the nucleosides A, T, or 5-MeC (1972-1974). 
They demonstrated that the absence of human ribosomal RNA and lack of silver staining of the remaining human NORs in mouse-human hybrid cells is due to the loss of one or more non-acrocentric human chromosomes, the first evidence of species-specific rRNA transcription factors. They confirmed this with Dr. Carlo Croce by showing that in rodent-human 'reverse' hybrids that tend to lose rodent chromosomes only human NORs are silver stained and only human $28 \mathrm{~S}$ rRNA is transcribed (1976-1977). The Miller group discovered, in two cancer cell lines and as a normal human variant, homogeneously staining regions (HSRs) containing amplified ribosomal DNA. In all of these cases, most of the amplified rRNA gene copies had been inactivated by massive DNA methylation (1981). Dr. D.A. Miller led comparative studies on great ape chromosomes and their evolution (1977), studies on chromosome-specific human and mouse satellite DNA sequences (1990, 1991), and comparative mapping of human and a marsupial by in situ hybridization with human gene probes (1994).

Jack served on the editorial board of Cytogenetics (19611972), American Journal of Human Genetics (1969-1974 and 1980-1983), Human Genetics (1978-1998), and Chromosome Research (1994-1997). He was associate editor of Cytogenetics and Cell Genetics (1972-1996) and of Genomics
(1987-1993). He served on scientific advisory committees for the National Foundation - March of Dimes (1967-present), the American Cancer Society (1974-1978 and 1986-1990), and the NIH (most recently, 1991-1994, on the Human Genome Study Section). Sandy served on the editorial board of Cytogenetics (1978-1984 and 1992-1996) and was an ad hoc reviewer for more than a dozen other journals.

Jack was Professor of Obstetrics and Gynecology and of Human Genetics and Development at Columbia University from 1969 to 1985. In 1982 he was certified in both Cytogenetics and Medical Genetics by the founding American Board of Medical Genetics and served as President of the ABMG in 1985 and 1986. In 1985 the Millers founded the Department of Molecular Biology and Genetics at Wayne State University School of Medicine in Detroit, Michigan, he serving as Chair and she as Professor. In 1983 Sandy had a sabbatical at the MRC Clinical and Population Cytogenetics Research Unit, Edinburgh, UK. She was Visiting Professor, Department of Genetics and Molecular Biology, University of Rome 'La Sapienza' in 1988 with Professor A. de Capoa and Distinguished Visiting Fellow in the Department of Genetics and Variation, LaTrobe University, Australia in 1991 with Professor Jennifer Marshall Graves. Jack and Sandy retired in 1996, but continue to periodically attend and contribute to scientific meetings. 


\title{
Program
}

Thursday, April 10

2:30-5:30 pm

ACC Board Meeting, Executive Board Room

2:30-7:00 pm

Conference Registration, Elevator Foyer

5:30-7:00 pm

Opening Reception, Portola Room

Sponsored by Agilent Technologies

Friday, April 11

7:00 am-12:00 pm

Conference Registration, Elevator Foyer

\section{Exhibitors are located in the Bonsai Ballroom: \\ Friday 7:00 am-Saturday 1:00 pm}

7:00-9:00 am

Continental Breakfast, Bonsai Ballroom

Sponsored by Quest Diagnostics

\author{
All Scientific Sessions will take place in the De Anza I Conference Room \\ 7:55-8:00 am \\ Welcome Address: Lauren Jenkins - President \\ Scientific Session 1: Constitutional Cytogenetics \\ Moderators: Susan Olson and Ankita Patel \\ 8:00-8:10 am 1 Molecular, Clinical and Cytogenetic Characterization of a Newly Described Microdeletion Syndrome at 22q11.2 \\ That Involves the Loss of the BCR Gene \\ L. Jenkins, E. Chen, K. Tezcan and A. Slavotinek \\ 8:10-8:20 am \\ 2 Complexity of 22q11 Rearrangements Detected by Oligonucleotide Microarray \\ 8:20-8:30 am \\ A.G. Mitchell, G. Richard, J. Dorson, J.G. Compton and S. Aradhya \\ 3 Three Cases of dup17p Revealed by CGH Microarray \\ B.R. DuPont, B. Griggs, A. Hunter, R. Schroer and M. Lyons \\ 8:30-8:40 am $\quad 4$ Terminal 12p Deletion in a Newborn and the Overlap of Clinical Features Previously Reported in This \\ Rare Deletion \\ J. LeRoux, K. Swisshelm, H. Rost, M. Springer and M. Kohn \\ $8: 40-8: 50 \mathrm{am}$ \\ 5 Interstitial Xp22.31 Duplication Detected by Array CGH in a Newborn with Congenital Heart Defect and \\ Dysmorphic Features \\ F. Li*, M. Palmquist, S.A. Morsey, J. Biscoe, E. Squibb, J. Chinsky and D.A.S. Batista \\ * Student Award Recipient \\ 8:50-9:00 am \\ 6 Karyotype-Phenotype Correlation for Down Syndrome: A Study of Mosaicism for Trisomy 21 \\ P. Papavassiliou*, T. York, U.T. Sundaram, N. Gursoy, G. Hill, B. Riley and C. Jackson-Cook \\ * Student Award Recipient \\ 9:00-9:10 am \\ Question \& Answer (Q \& A)
}

Preprint Cytogenet Genome Res 121/1/2008

Abstracts will be available online,

free of charge 
7 Expression of Phenotype in a Diploid Genome

Invited Speaker: Steve Scherer, Ph.D.

The Centre for Applied Genomics, Hospital for Sick Children and University of Toronto

Introduced by Daynna Wolff

Sponsored by GeneDx

Coffee Break - Bonsai Ballroom

Sponsored by Signature Genomic Laboratories

\section{Scientific Session 2: Cancer Cytogenetics}

Moderators: Urvashi Surti and Catherine Rehder

8 MLL Amplification in Acute Leukemia: Including One Case of T-ALL

D.I. Quigley and D.J. Wolff

9 Amplification of $A B L 1$ in T-Cell Acute Lymphoblastic Leukemia: Report of a New Case and Review of the Literature A.M. Meloni-Ehrig, J. Jahn, J. Scheerle, S. Revis, J. Zgoda, T. Tchen, D.A. Cipkala, L.J. Ettinger, E.S. Gamboa, J. Kelly, J. Meck, N. Christacos and P.N. Mowrey

10 Minimally Differentiated Acute Myeloid Leukemia (AML-M0) and $\mathbf{t}(8 ; 14)$ with Single IGH-CMYC Fusion on the der(8) G.V.N. Velagaleti, C. Holladay, J. Youhas, R. Wang, K. Lundberg, D. Cherry and R.P. Ketterling

11 Two New Cases of Translocation Involving NUP98 in AML Patients

H. Bruyère, D.E. Horsman, K. Chipperfield, D. Forrest, M. Hudoba de Badyn, K.W. Song and R.K. Humphries

12 Segmental Jumping Translocations: A Review of a Rare Rearrangement Involved in Clonal Evolution and Disease Progression in Leukemias and Lymphomas

P. Singh-Kahlon and C.F. Stephenson

13 Frequency of 5'IGH Deletions in B-Cell Chronic Lymphocytic Leukemia/Small Lymphocytic Lymphoma (CLL/SLL) F. Nooraie, F. Quintero-Rivera and P.N. Rao

14 Chronic Lymphocytic Leukemia (CLL) Patients with High-Risk Cytogenetics Respond to Treatment with Flavopiridol: Study of 120 Patients (pts) with Relapsed, Genetically High Risk CLL

N.A. Heerema, T.S. Lin, B. Fischer, G. Lozanski, K.A. Blum, L.A. Andritsos, J.A. Jones, J.M Flynn, M.E. Moran, S.M. Mitchell, L.J. Schaaf, A.J. Johnson, L.L. Smith, A.J. Wagner, C.A. Raymond, M. Phelps, J.T. Dalton, M.R. Grever and J.C. Byrd

15 Idiopathic Myelofibrosis with Ring Chromosome 10 Leading to Deletion of PTEN in a 21 Month Old Female

J. Kelly, A.M. Meloni-Ehrig, J. Jahn, J. Scheerle, L. Matyakhina, M. Motz, N. Shahabazi, J. Ramdas, K. Zhang, P. Dorion, J. Meck, N. Christacos and P.N. Mowrey

16 Black Tea (Camellia sinensis) as a Chemopreventive Agent in Oral Leukoplakia

A. Halder, R. Raychowdhury, U. Das and M. De

$\mathbf{Q} \& \mathbf{A}$

Lunch on Your Own

\section{Scientific Session 3: FISH Methodology}

Moderators: Arthur Brothman and Yassmine Akarri

Harold Klinger Memorial Lecture

17 The Evolutionary History of Mammalian Genome Organization

Invited Speaker: Johannes Wienberg, Ph.D.

Introduced by Peter Jacky

Sponsored by Karger Publishers

18 Classification of Multiple Myeloma into Prognostic Groups Based on Karyotype and FISH

H.A. Aviv, I. Maxwell, J. Wang, K. Vassallo, A. Ninan, C. Creese and D. Ang

19 Improved Detection of Chromosome Abnormalities in Patients with MDS Utilizing Interphase FISH

M.S. Matthews

20 Deletions on Derivative Chromosome 9 in CML with $\mathrm{t}(9 ; 22)$ Arise Not Only when the Translocation Initially Occurs, but also as Part of the Clonal Evolution - Evidence from More than 50 Cases

N.S. Mitter

21 Unexpected Complexity of Supernumerary Marker Chromosomes Revealed by Microarray Comparative Genomic Hybridization

K.D. Tsuchiya, K. Opheim, M. Hannibal, A. Hing, I.A. Glass, M. Raff, C. Beattie, T. Norwood and B. Torchia 


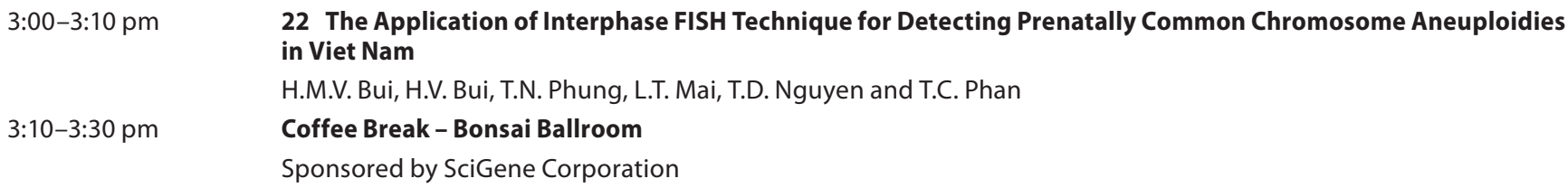

\section{Scientific Session 4: FISH Validation and Discussion}

Moderator: Robert Best and Sarah South

3:30-3:40pm

$3: 40-3: 50 \mathrm{pm}$

3:50-4:00 pm

4:00-5:00 pm

5:00-7:00 pm
23 Validation of Fluorescence in situ Hybridization Assays: BETAINV versus Mean plus 3 Standard Deviations for Calculation of Normal Cut-Off Values

L.R. Rowe, L.S. Rector, A.N. Lamb, A.R. Brothman and S.T. South

24 Assessment and Validation of the BioView Duet System for Automated Scoring of UroVysion

D.J. Wolff, R. Rogers and D.I. Quigley

25 A Statistical Method for Calculating FISH Reference Ranges over a Large Number of Values

A.L. Ciolino, M.E. Tang and R.J. Bryant

Discussion of FISH Validations

Free Time - Yeah!

\section{Distinguished Cytogenetics Award Dinner Takes Place in the De Anza I Ballroom \\ 7:00-10:00 pm \\ $8: 30-8: 45 \mathrm{pm}$ \\ 8:45-9:15 pm \\ 9:15-10:00 pm \\ $10: 00 \mathrm{pm}$ \\ ACC 2008 Distinguished Cytogeneticist Award Dinner \\ Award Recipients: Dr. Orlando J. Miller and Dr. Dorothy Anne Miller \\ Introduction: Arthur Brothman \\ Discussion of Career and Accomplishments \\ Award Presentation and Remarks \\ Session Closing}

Saturday, April 12

7:00-8:30 am

7:00-12:30 pm

7:30-8:00 am

8:00-8:10 am

8:10-8:20 am

8:20-8:30 am

8:30-8:40 am

$8: 40-8: 50$ am

8:50-9:00 am

9:00-9:10 am

\section{Continental Breakfast - Bonsai Ballroom}

Sponsored by Baylor College of Medicine Medical Genetics Laboratories

Conference Registration - Elevator Foyer

ACC Business Meeting

With Student Award Presentations

\section{Scientific Session 5: Constitutional Cytogenetics}

Moderators: Peter Jacky and Anne Higgins

26 Prenatal Diagnosis of a de novo Unbalanced X-Autosome Translocation by Array-CGH in a Female Fetus with an Increased Nuchal Translucency

E. Kolomietz, J. Mazurkiewicz and D. Chitayat

27 Evaluation of Anencephaly, Rachischisis, and Omphalocele in a Stillborn Cynomolgus Monkey (Macaca fascicularis)

S.M. Gardner, C.M. Moore, E.J. Dick, G.B. Hubbard, B.G. Dunn, V. Williams, C. Keller and M.D. Davis

28 Update on Placental Mesenchymal Dysplasia, Chimeras, Mosaics and P57 ${ }^{\text {KIP2 }}$

U. Surti and L. Hoffner

29 No Association of Skewed X Inactivation with Recurrent Abortion or Trisomy

D. Warburton, J. Kline, S. Brown, A. Morin, C.-Y. Yu and B. Levin

30 Sex Chromosome Loss in Lymphocyte Culture: Incidental or Clinically Relevant Finding; When Do You Report It? S. Shetty and J.E.Chernos

31 Cytogenetic Study of 245 Infertile Men in Taiwan: Secret Behind the Number?

M.H. Cheng, P.H. Wang, M.J. Yang, H.T. Chao, S.P. Chang, K.C. Chao and J.S. Huang

Q \& A 


\section{Scientific Session 6: Array CGH}

Moderators: Hutton Kearney and Lauren Jenkins

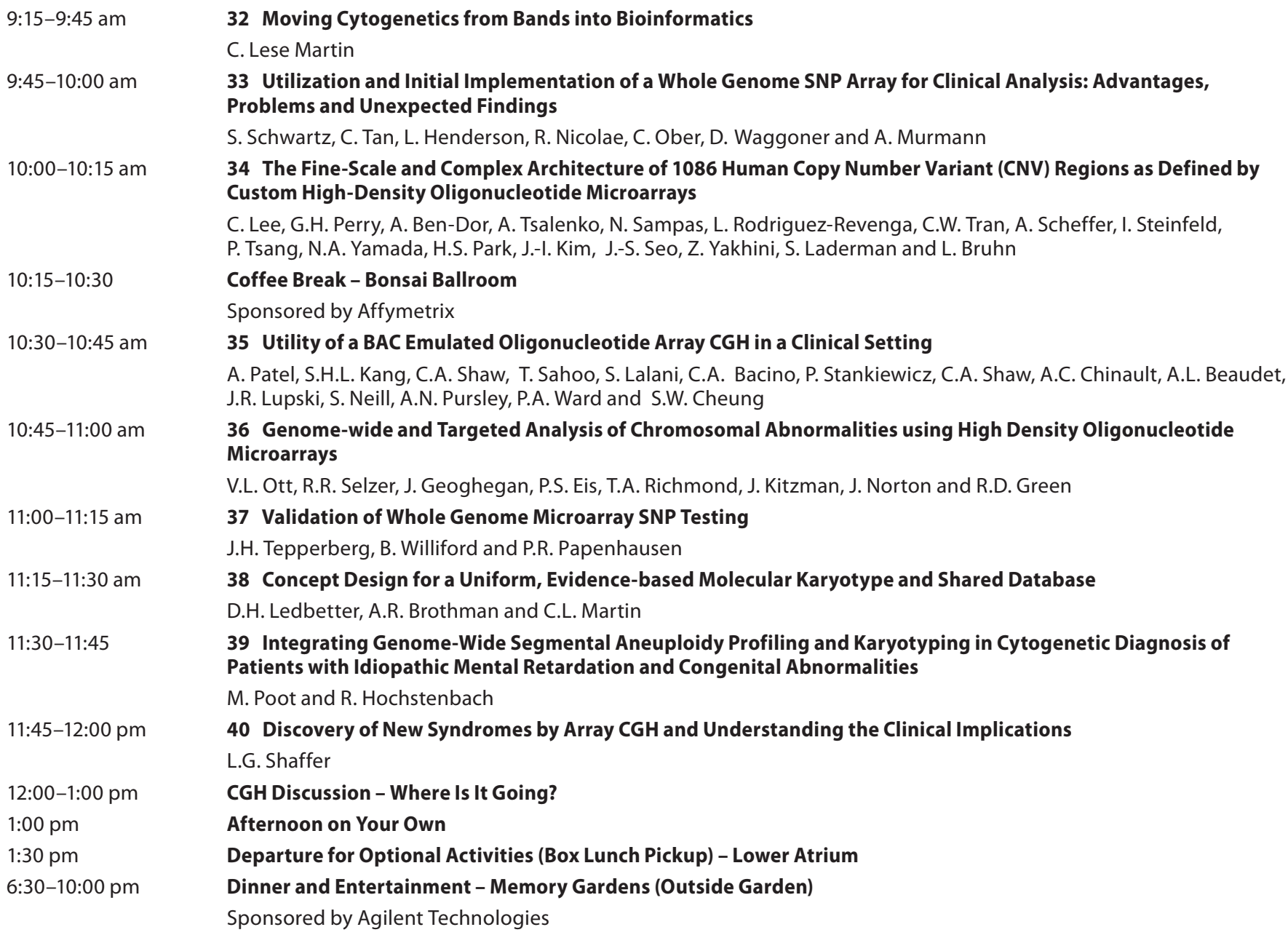

\section{Scientific Session 7: Cytogenetics}

Moderators: Athena Cherry and Jennifer Winters

41 Laboratory Quality Improvement and Financial Success through Six Sigma Methodology S.M. Zneimer

42 Is Unreported Chromosomal Variation in Hyraxes Indicative of Cryptic Speciation?

M.L. Houck, S.J. Charter, L.G. Chemnick, H.A. Davis, J.A. Fronczek, A. Johnson and O.A. Ryder

43 Chromosomal Aberrations Associated with Congenital Abnormalities, Infertility, and Repeated Early Embryonic Loss in the Domestic Horse (Equus caballus)

T.L. Lear and J. Lundquist

44 Experience with a Microarray Designed Specifically to Characterize Marker Chromosomes C.D. Kashork, B.C. Ballif and L.G. Shaffer

45 Novel Genomic Syndromes Defined by Array CGH Are Focused in Regions of High Complexity S. Aradhya, A.G. Mitchell, S. Warren, J. Dorson and G. Richard 46 Expansion in Size of a Terminal Deletion: A Paradigm Shift for Parental Follow-up Studies S.T. South, A.F. Rope, A.N. Lamb, E. Aston, N. Glaus, H. Whitby, T. Maxwell, X.L. Zhu and A.R. Brothman Q \& A 


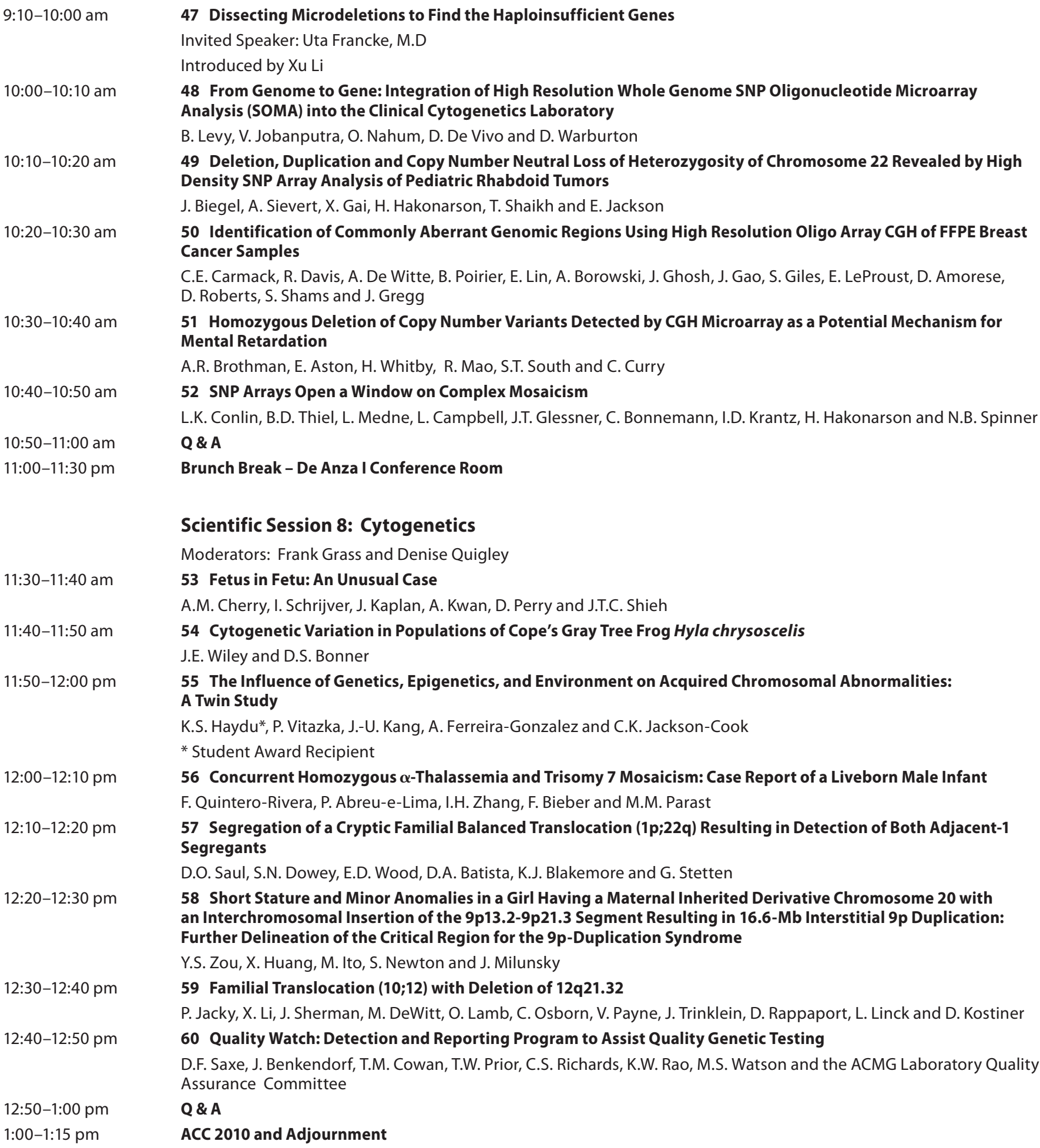

47 Dissecting Microdeletions to Find the Haploinsufficient Genes Invited Speaker: Uta Francke, M.D

Introduced by Xu Li

48 From Genome to Gene: Integration of High Resolution Whole Genome SNP Oligonucleotide Microarray Analysis (SOMA) into the Clinical Cytogenetics Laboratory

B. Levy, V. Jobanputra, O. Nahum, D. De Vivo and D. Warburton

49 Deletion, Duplication and Copy Number Neutral Loss of Heterozygosity of Chromosome 22 Revealed by High Density SNP Array Analysis of Pediatric Rhabdoid Tumors

J. Biegel, A. Sievert, X. Gai, H. Hakonarson, T. Shaikh and E. Jackson

50 Identification of Commonly Aberrant Genomic Regions Using High Resolution Oligo Array CGH of FFPE Breast Cancer Samples

C.E. Carmack, R. Davis, A. De Witte, B. Poirier, E. Lin, A. Borowski, J. Ghosh, J. Gao, S. Giles, E. LeProust, D. Amorese, D. Roberts, S. Shams and J. Gregg

51 Homozygous Deletion of Copy Number Variants Detected by CGH Microarray as a Potential Mechanism for Mental Retardation

A.R. Brothman, E. Aston, H. Whitby, R. Mao, S.T. South and C. Curry

52 SNP Arrays Open a Window on Complex Mosaicism

L.K. Conlin, B.D. Thiel, L. Medne, L. Campbell, J.T. Glessner, C. Bonnemann, I.D. Krantz, H. Hakonarson and N.B. Spinner Q \& A

Brunch Break - De Anza I Conference Room

\section{Scientific Session 8: Cytogenetics}

Moderators: Frank Grass and Denise Quigley

53 Fetus in Fetu: An Unusual Case

A.M. Cherry, I. Schrijver, J. Kaplan, A. Kwan, D. Perry and J.T.C. Shieh

54 Cytogenetic Variation in Populations of Cope's Gray Tree Frog Hyla chrysoscelis

J.E. Wiley and D.S. Bonner

55 The Influence of Genetics, Epigenetics, and Environment on Acquired Chromosomal Abnormalities: A Twin Study

K.S. Haydu*, P. Vitazka, J.-U. Kang, A. Ferreira-Gonzalez and C.K. Jackson-Cook

* Student Award Recipient

56 Concurrent Homozygous $\alpha$-Thalassemia and Trisomy 7 Mosaicism: Case Report of a Liveborn Male Infant F. Quintero-Rivera, P. Abreu-e-Lima, I.H. Zhang, F. Bieber and M.M. Parast

57 Segregation of a Cryptic Familial Balanced Translocation (1p;22q) Resulting in Detection of Both Adjacent-1 Segregants

D.O. Saul, S.N. Dowey, E.D. Wood, D.A. Batista, K.J. Blakemore and G. Stetten

58 Short Stature and Minor Anomalies in a Girl Having a Maternal Inherited Derivative Chromosome 20 with an Interchromosomal Insertion of the 9p13.2-9p21.3 Segment Resulting in 16.6-Mb Interstitial 9p Duplication: Further Delineation of the Critical Region for the 9p-Duplication Syndrome

Y.S. Zou, X. Huang, M. Ito, S. Newton and J. Milunsky

59 Familial Translocation (10;12) with Deletion of 12q21.32

P. Jacky, X. Li, J. Sherman, M. DeWitt, O. Lamb, C. Osborn, V. Payne, J. Trinklein, D. Rappaport, L. Linck and D. Kostiner 60 Quality Watch: Detection and Reporting Program to Assist Quality Genetic Testing

D.F. Saxe, J. Benkendorf, T.M. Cowan, T.W. Prior, C.S. Richards, K.W. Rao, M.S. Watson and the ACMG Laboratory Quality Assurance Committee

Q \& A

ACC 2010 and Adjournment 


\section{1}

\section{Molecular, Clinical and Cytogenetic Characterization of a Newly Described Microdeletion Syndrome at 22q11.2 That Involves the Loss of the BCR Gene}

\section{Jenkins ${ }^{a}$, E. Chen ${ }^{a}$, K. Tezcan ${ }^{a}$, A. Slavotinek ${ }^{b}$}

aThe Permanente Medical Group, Inc. Genetics Department, Northern California, ${ }^{b}$ Department of Genetics, Division of Genetics, University of California, San Francisco, CA, USA

The emergence of whole genomic microarrays (aCGH) has resulted in the identification of new contiguous gene syndromes that were previously undetectable using high-resolution cytogenetic analysis. One such microdeletion syndrome at 22q11.2 was fortuitously identified by the use of the subtelomeric panel of probes from Abbott/Vysis Inc (Ravnan et al., 2006. J Med Genet 43:478-489). Several individuals were found to have a deletion of the $B C R$ gene, which serves as an internal control in the mix containing probes specific to the subtelomeric region at 22 qter. The estimated incidence of the deletion was $8 / 11,688$ patients with learning disabilities or a frequency of $0.07 \%$, but minimal clinical information was available. Systematic molecular analysis of patients with features of velocardiofacial syndrome (VCFS) also has identified a few rare cases with 'atypical deletions' in the 22q11.2 region that are adjacent but do not overlap with the known deletions for VCFS (Rauch et al., 2005. J Med Genet 42:871-876; Saitta et al., 1999. 65: $562-566)$. Recently, the clinical and molecular findings of one individual with a $B C R$ deletion has been reported (Mikhail et al., 2007. Am J Med Genet Part A 143A:2178-2184). We report on two additional, unrelated individuals with submicroscopic deletions at 22q11.2 that include the loss of the $B C R$ locus. Case 1 is a 10 year old Filipino boy with mildly dysmorphic facial features, bilateral cleft lip and palate, preauricular tags and dysplastic ears, ventricular septal defect, patent ductus arteriosus, inguinal hernia, severe articulation disorder, mild mental retardation and developmental delay. Case 2 is a 9 year old male with bilateral 'forme fruste' cleft lip and cleft alveolar notch, microcephaly, and mild facial dysmorphism which includes mildly upslanting palpebral fissures and synophrys, a broad nasal bridge with anteverted nares, and ear dysplasia. He had a slight kyphosis at the cervicothoracic junction, relative brachydactyly of his fingers, valgus deviation of the halluces, pes planus, obesity and mild developmental delay. The deletions were mapped using a $44 \mathrm{~K}$ whole genome oligonucleotide microarray from Agilent, Inc. Case 1 was found to have a deletion of 2.4 $\mathrm{Mb}$ (from nucleotide 20,312,379 to 22,714,231), with a loss of 37 genes and case 2 with a deletion of $2.8 \mathrm{Mb}$ (from nucleotide 20,128,705 to $22,961,613)$, and a loss of 43 genes. This region is just telomeric to the DiGeorge/Velocardiofacial critical region and is flanked by low copy repeat (LCR) regions that may play a role in non-allelic homologous recombination (NAHR). Both patients described have orofacial clefts, suggesting that testing for this deletion may be important in the evaluation of children with cleft lip/palate, mild dysmorphism and developmental disabilities. Assessment of the clinical and molecular findings of a collection of these patients will be presented to delineate the common features observed in this contiguous gene syndrome.

\section{2 \\ Complexity of 22q11 Rearrangements Detected by Oligonucleotide Microarray}

A.G. Mitchell, G. Richard, J. Dorson, J.G. Compton, S. Aradhya

GeneDx

Misalignment of low-copy repeat (LCR) sequences in chromosome $22 \mathrm{q} 11$ predisposes this region to genomic rearrangement. 1.5-3.0 Mb deletions are commonly associated with the 22q11 microdeletion syndrome including DiGeorge/Velocardiofacial syndrome (DG/VCFS), while reciprocal duplications of similar size have been recently described in the '22q11.2 duplication syndrome'. Using oligonucleotide microarray (oligoarray), we have identified 15 cases with 22q11 genomic imbalance, of which only $1 / 3$ were deletions located within the critical region (CR) encompassing the TBX1 and HIRA (TUPLE1) genes. Five atypical deletions associated with variable phenotypes were detected ranging in size from $680 \mathrm{~kb}$ to $3.0 \mathrm{Mb}$. One deletion located in the very distal portion of the CR (and not including the TBX1 gene) was found in a child with developmental delay, cleft lip and/or palate, microcephaly, a congenital heart defect, and diaphragmatic hernia. The other 4 atypical deletions were positioned distal (telomeric) to and not overlapping with the CR. One patient diagnosed with a cerebellopontine angle (CPA) tumor (atypical teratoid rhabdoid tumor of the brain) harbored a distal, non-overlapping deletion which included SMARCB1, a tumor suppressor gene implicated in malignant rhabdoid tumors (MRT), atypical teratoid tumors, and familial posterior fossa tumors of infancy. The remaining 5 cases involved clinically affected patients with genomic gains in the 22q11 region including two duplications located distal to and not overlapping with the $\mathrm{CR}$, and two duplications overlapping with the proximal portion of the CR. Finally, a quadruplication of the DG/VCFS region was identified in a child with developmental delay who inherited one copy of the duplication from each phenotypically-normal parent. 3 of $5(60 \%)$ duplications were reportedly not found by prior FISH 22q11 studies. Overall, 6/10 (60\%) rearrangements occurred de novo and $4 / 10(40 \%)$ were inherited; the inheritance pattern of the remaining 5 cases is unknown. From these results emerge two significant findings. In our cohort, 22q11 duplication events represented $1 / 3$ of the total rearrangements and were not uncommon. We noted clinical diversity among these patients, an observation that supports previous publications on the 22q11 duplication phenotype. Secondly, the majority $(2 / 3)$ of patients presented with atypical deletions/ duplications outside of the 22q11 CR that would go undetected by conventional cytogenetics. Therefore, high density array is a valuable tool to identify the complete spectrum of $22 \mathrm{q} 11$ genomic rearrangements and help refine the phenotypes associated with both duplications and atypical deletions. 


\section{3}

\section{Three Cases of dup17p Revealed by CGH Microarray}

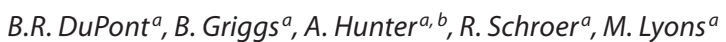

${ }^{\mathrm{a}}$ Greenwood Genetic Center, Greenwood, SC, USA; ${ }^{b}$ Children's Hospital of Eastern Ontario, Canada

Over the past few years, CGH microarray analysis has been utilized more frequently by many clinical cytogenetics laboratories and their physicians. This analysis has become a viable tool for clinical analysis of patients with disorders of unknown etiology and for confirmation of known clinical entities. We report here the finding of three patients with duplications of chromosome 17p. Patient A is a 6 year old female who presented with muscle weakness, areflexia and decreased sensation, findings which were consistent with Charcot-Marie-Tooth disease. There was a positive family history indicating an autosomal dominant form of the disease. Standard karyotype analysis was not performed. CGH targeted microarray revealed an approximately $1 \mathrm{Mb}$ duplication of $17 \mathrm{p} 12$, which includes the PMP22 gene, and is consistent with Charcot-Marie-Tooth 1A duplication. Patient B is a 3 year old male who has microcephaly, undergrowth, global developmental delay and dystonia. Routine chromosome analysis was normal. CGH targeted microarray revealed an approximately $1 \mathrm{Mb}$ duplication of $17 \mathrm{p} 12$, this duplication also includes the PMP22 gene. However, his history and clinical findings are not consistent with CMT $1 \mathrm{~A}$. Patient $\mathrm{C}$ is a 5 year old male who was evaluated for possible FG syndrome. Previous routine chromosome analysis at another institution was normal. CGH targeted microarray revealed a $4 \mathrm{Mb}$ duplication of $17 \mathrm{p} 11.2$. This duplication includes part of the SMS region. These results support the use of CGH microarray analysis for both clinical confirmation studies and on patients with disorders of unknown etiology.

\section{4 \\ Terminal 12p Deletion in a Newborn and the Overlap of Clinical Features Previously Reported in This Rare Deletion \\ J. LeRoux ${ }^{a}$, K. Swisshelm ${ }^{a}$, H. Rost ${ }^{a}$, M. Springer ${ }^{a}$, M. Kohn ${ }^{b}$ \\ ${ }^{a}$ Colorado Genetics Laboratory, ${ }^{b}$ University of Colorado Health \\ Sciences Center, Denver, CO, USA}

A newborn female was referred for dysmorphic facies, simple ears and double manubrium ossification centers. The cytogenetic karyotype suggested a deletion at $12 \mathrm{p} 13.31$ with a terminal deletion confirmed by subtelomere and ETV6 FISH studies. The reported 12p deletions are more often interstitial or result from a parental rearrangement. Parental cytogenetic and subtelomere FISH studies in this case were normal, indicating a rare de novo event.

Monosomy for distal $12 \mathrm{p}$ is uncommon and the phenotypic features are varied. Overlap of clinical findings with our patient included craniofacial dysmorphology, specifically manifesting as simple ears, and skeletal/chest malformations, which present here as the double manubrium ossification centers. One of the genes located within the subtelomeric region is the WNT5B which is involved in growth regulation, and which may contribute to the phenotypic effects seen with this deletion.

\section{5 \\ Interstitial Xp22.31 Duplication Detected by Array CGH in a Newborn with Congenital Heart Defect and Dysmorphic Features}

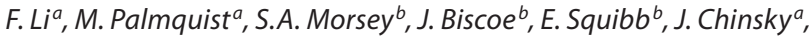 \\ D.A.S. Batistab,c
}

a McKusick-Nathans Institute of Genetic Medicine, ${ }^{\text {b }}$ Cytogenetics Laboratory, Kennedy Krieger Institute, 'Department of Pathology, Johns Hopkins University, Baltimore, MD, USA

A number of microdeletion and microduplication syndromes are caused by non-allelic homologous recombination (NAHR) between segments of low-copy repeats. In some individuals with X-linked ichthyosis (XLI), NAHR of regions flanking the STS gene results in a recurrent microdeletion at Xp22.31. Mental retardation might or not be present in XLI, probably depending on the extent of and specific segment deleted. There are at least five other genes in this $\sim 2 \mathrm{Mb}$ region: VCX3A, HDHD1A, VCX, PNPLA4, and VCX2. Deletion of VCX3A has been implicated in mental retardation in some cases; however other individuals lacking this gene are not cognitively impaired. A similar microduplication of this region of Xp22.31 has been reported in very few individuals. Here we report a newborn boy with congenital hypoplastic left heart and other dysmorphic features with likely NAHR of Xp22.1. He was born at 38 5/7 weeks to a G1P0 mother. The APGARS were 4, 6, and 8 at 1, 5 and $10 \mathrm{~min}$, respectively. The birth weight and height were above the $50^{\text {th }}$ percentile, and the head circumference was between the $25^{\text {th }}$ and $50^{\text {th }}$ percentiles. At the time of birth, he had no respiratory effort and was intubated. Physical examination showed epicanthal folds, hypertelorism, a broad nose with anteverted nares, a barrel shaped chest, transverse palmar creases, a deep sacral dimple, short fingers with $5^{\text {th }}$ finger clinodactyly and $4^{\text {th }}-5^{\text {th }}$ toe syndactyly. The boy's karyotype was 46,XY. FISH ruled out a 22q11 deletion (VCFS). A high resolution BAC-array CGH with 4,200 clones detected gain of eight clones at Xp22.31 (from RP11-483M24 to RP11-351B15). The gain was estimated to be between $1.1 \mathrm{Mb}$ and $2.2 \mathrm{Mb}$. This is the first case reported of interstitial duplication of Xp22.31 associated with major congenital heart defect. Further studies are in process to delineate the extent of the microduplication and to understand its genetic mechanism.

\section{6 \\ Karyotype-Phenotype Correlation for Down Syndrome: A Study of Mosaicism for Trisomy 21}

P. Papavassiliou ${ }^{a}$, T. York ${ }^{a, b}$, U.T. Sundaram ${ }^{a}$, N. Gursoy $^{a, d}$, G. Hill ${ }^{a, e}$, B. Riley ${ }^{a, b}$, C. Jackson-Cook ${ }^{a, c}$

Departments of a Human Genetics, cPsychiatry and bPathology, Virginia Commonwealth University, Richmond, VA, ${ }^{\mathrm{d} D e p a r t m e n t}$ Neurology, Cleveland Clinic Foundation, Cleveland, $\mathrm{OH}$,

eForensic Biology Section, Virginia Department of Forensic Science, Eastern Laboratory, Norfolk, VA, USA

Little is known about the pathogenesis of the phenotype of mosaic Down syndrome (MDS). Thus, the primary goal of this study was to identify factors contributing to phenotypic variation by studying 107 individuals having MDS from 8 different countries. To investigate a potential 'threshold' effect due to trisomic imbalance, lymphocyte and buccal mucosa nuclei were scored using FISH. Overall, buccal cells had significantly higher frequencies of trisomy (mean \pm s.e., $46.06 \pm$ $3.44 \%)$ than lymphocytes $(30.13 \pm 3.99 \%)(\mathrm{p}<0.0001)$. Also, diagnostic analyses showed higher trisomic percentages than assessments at later ages, suggesting a possible selection against trisomic lymphocytes over time $(\mathrm{p}<0.0001)$. Using latent class analysis, two phenotypic 'classes' were identified. The observation of a significantly increased percentage of trisomic cells in the probands from the class having more 
traits (class 2) compared to the class with fewer traits (class 1) suggested that the percentage of mosaicism in a patient does influence the phenotypic outcome. Tissue-specific influences were also detected, with buccal mucosa trisomy levels being significantly correlated with IQ ( $p=0.0094)$ (both ectodermal derivatives), while congenital heart defects were significantly associated with lymphocytes $(\mathrm{p}=0.0286)$ (both mesodermal). Phenotypic influences due to the nondisjunction$\mathrm{al} /$ recombination event(s) resulting in mosaicism were also seen, with significantly more traits being observed in probands having 2 copies of an identical allele(s) $(\mathrm{MII} /$ mitotic errors) $(\mathrm{p}=0.03)$. However, the majority (35/37) of mosaic cases arose from two nondisjunctional errors (meiotic and mitotic). The potential impact of trisomy 21 on telomere length was evaluated in the isogenic trisomic and euploid cells of mosaic probands using a semi-quantitative FISH assay. For each individual, a significant difference in telomere lengths was observed in the trisomic compared to euploid cells. Also, significantly longer telomeres were seen in the trisomic cells from the youngest probands (5 months to 2 years), with significantly shorter telomeres being seen in the trisomic cells from the oldest (11 years) proband. In conclusion, variation in phenotype (allowing for the distinction of two classes) was influenced by the percentage of trisomic cells (with tissue-specific effects being observed), as well as the chromosomal malsegregation/recombination error(s) that gave rise to the mosaicism. Furthermore, the impact of a trisomic imbalance for chromosome 21 was observed on a cellular (telomere lengths) as well as an organismal level. The results of this study should provide geneticists and other health care professionals with more complete information regarding optimal diagnostic approaches, anticipated clinical outcomes, and recurrence risks for families having a child with MDS.

\section{7}

\section{Expression of Phenotype in a Diploid Genome}

\section{S.W. Scherer}

The Centre for Applied Genomics, Hospital for Sick Children and University of Toronto, Canada

Variation in human DNA takes many forms, but it is only recently that robust technologies have become available that allow genomewide surveys of structural variation, in particular for copy number variation $(\mathrm{CNV})$. Our group is interested in understanding the impact structural variation has on phenotypes and aims to generate a chromosome imbalance map of the diploid human genome. As such, we have used microarray, sequencing, cytogenomic, and data mining technologies to identify thousands of structural variations in genomes from the general population. They are collated with all other similar public information in the 'Database of Genomic Variants'. We have also examined thousands of genomes from individuals with defined traits or disease to examine how structural variants might influence the expression of phenotype, most notably by altering gene dosage away from a typical diploid status. One of the major focuses of our work has been studying structural variation of chromosomes in autism spectrum disorder. Our collective findings reveal a somewhat surprising level of $\mathrm{CNV}$ in the human genome that is tolerable in certain chromosomal regions, and not in others. In between, there is a 'grey-zone' where the effect can be modified by the other allele, copy number, gender, parental inheritance, and other variants. Ultimately, it is the interplay of all of these that contributes to the continual evolution of humans.

The objectives of this presentation are to: (1) provide an update on the current literature regarding CNVs and inversions and their impact on disease, (2) identify and discuss the limitations in our understanding in this new field (such as new mutation rate, structural variation in somatic cells, etc.), and (3) discuss new technologies including higher resolution microarrays, $\mathrm{CNV}$ prediction algorithms, and whole-genome sequencing approaches.

\section{MLL Amplification in Acute Leukemia: Including One Case of T-ALL}

\section{D.I. Quigley, D.J. Wolff}

Department of Pathology and Laboratory Medicine,

Medical University of South Carolina, Charleston, SC, USA

The mixed lineage leukemia (MLL) locus has been shown to be altered in multiple hematological diseases: myelodysplastic syndromes (MDS), both myeloid and lymphoid acute leukemias and treatment related leukemias. While the majority of MLL abnormalities involve rearrangements resulting in novel fusion proteins with oncogenic activity, another mechanism for MLL oncogenic activity is via gene amplification. MLL amplification has primarily been reported in MDS and AML and is associated with advanced patient age and poor prognosis. Amplification of MLL presents by various cytogenetic findings including double minute (dmin) chromosomes, large derivative chromosomes with homogeneously staining regions (HSRs) and rings or marker chromosomes. We present four cases: three cases of AML with complex karyotypic findings and MLL amplification by FISH and one case with T-cell ALL. Two AML cases showed MLL amplification within HSRs in large derivative chromosomes 11 and concomitant deletion of 5q. The third case with AML showed MLL amplification within dmin chromosomes. Each of these individuals had rapidly progressive disease and short survival. The case with T-cell ALL, a 19 year old male patient, presented with a translocation $\mathrm{t}(10 ; 11)(\mathrm{p} 13 ; \mathrm{q} 21)$. FISH was performed to confirm that the translocation did not involve the MLL locus. Four copies of MLL were observed in about $20 \%$ of nuclei examined. The disease course was aggressive and the patient succumbed to disease 8 months after diagnosis. There is only one other case of MLL amplification in ALL, designated at pre-B lineage. To our knowledge this is the first report of MLL amplification in T-ALL.

\section{9 Amplification of ABL1 in T-Cell Acute Lymphoblastic Leukemia: Report of a New Case and Review of the Literature \\ A.M. Meloni-Ehrig a , J. Jahn ${ }^{a}$, J. Scheerle ${ }^{a}$, S. Revis ${ }^{a}$, J. Zgoda ${ }^{a}$ \\ T. Tchen ${ }^{a}$, D.A. Cipkala ${ }^{b}$, L.J. Ettinger ${ }^{b}$, E.S. Gamboa ${ }^{c}$, J. Kelly ${ }^{a}$, J. Meck ${ }^{a}$, N. Christacos ${ }^{a}$, P.N. Mowrey ${ }^{a}$ \\ aLaboratory of Cytogenetics, Quest Diagnostics Nichols Institute, Chantilly, VA, Departments of 'Pediatric Hematology-Oncology, 'Laboratory Medicine and Pathology, Saint Peter's University Hospital, New Brunswick, NJ, USA}

$A B L 1$ amplification, due to a cryptic episomal circular fusion of the genes NUP214 to $A B L 1$, both located at $9 \mathrm{q} 34$, is a new phenomenon in T-ALL and only a few cases have been reported. There is divergent opinion on which therapeutic approach to use in these cases. Some reports suggest that these patients appear to be initially sensitive to imatinib mesylate (Gleevec) and therefore, this treatment should be considered. However, it was shown that the same patients later acquire additional chromosome abnormalities and subsequent resistance to this drug $[1,2]$. There are also reports of cases with absolutely no response to Gleevec [3], thereby leaving questions about the utility of Gleevec in the treatment of these patients.

We present a case of a 6-year-old male with T-ALL who presented with several weeks of anorexia, nausea, and vomiting. At presentation, the physical examination revealed a shoddy bilateral cervical and supraclavicular lymphadenopathy. A chest $\mathrm{x}$-ray revealed a large anterior mediastinal mass with a slight left-sided pleural effusion. Laboratory studies of the peripheral blood revealed the following: WBC 7200 with 54 neutrophils, 29 lymphocytes, and 17 monocytes; platelets 157,000; and no peripheral blasts. His initial uric acid was $42 \mu \mathrm{g} / \mathrm{dl}$ with a cre- 
atinine of $3.5 \mu \mathrm{g} / \mathrm{dl}$. The bone marrow aspirate smear and biopsy revealed 53\% and 95\% blasts, respectively. By immunohistochemistry, the blasts were positive for the T-cell markers CD3 and CD43 and negative for the B-cell markers CD20 and CD79a. The morphologic and immunohistochemical findings were consistent with the diagnosis of T-ALL. Chromosome analysis revealed additional material on $7 p$ and a questionable rearrangement on $9 \mathrm{p}$. As such, FISH studies were performed with the p16 (9p21) probe, in addition to the BCR/ABL probe (Abbott Molecular/Vysis), which is required by the Children's Oncology Group (COG) protocol. FISH showed amplification of ABL1 (4-15 signals) as well as deletion of 9p21 in 26\% and 7\% of interphase cells examined, respectively. Upon confirmation of the diagnosis, induction chemotherapy was initiated with vincristine, daunomycin, prednisone, PEG-asparaginase, intrathecal cytarabine, and intrathecal methotrexate. The day- 8 bone marrow showed no evidence of leukemia, and the minimal residual disease on day 29 was negative by flow cy tometry. Standard consolidation therapy consisted of vincristine, cytarabine, cyclophosphamide, oral 6-mercaptopurine, PEG-asparaginase, and 4 doses of intrathecal methotrexate. The interim maintenance therapy consisted of 4 courses of vincristine, high-dose intravenous methotrexate with leukovorin rescue, daily oral 6-mercaptopurine, and 2 doses of intrathecal methotrexate,. The patient continues therapy and has been in complete remission for 7 months.

\section{References}

1 Bernasconi et al: ABL1 amplification in T-cell acute lymphoblastic leukemia. Cancer Genet Cytogenet 162:146-150 (2005)

2 Graux et al: Fusion of NUP214 to ABL1 on amplified episomes in T-cell ALL. Nat Genet 36:1086-1089 (2004).

3 Stergianou et al: Fusion of NUP214 to ABL1 on amplified episomes in T-cell ALL - implications for treatment. Leukemia 19:1680-1681 (2005).

\section{0 \\ Minimally Differentiated Acute Myeloid Leukemia (AML-M0) and $t(8 ; 14)$ with Single IGH-CMYC Fusion on the der(8) \\ G.V.N. Velagaleti ${ }^{a}$, C. Holladay ${ }^{b}$, J. Youhas $^{a}$, R. Wang ${ }^{a}$, K. Lundberg $^{a}$, D. Cherry ${ }^{b}$, R.P. Ketterling ${ }^{a}$ \\ ${ }^{a}$ Cytogenetics Laboratory, Mayo Clinic, Rochester, MN, \\ 'DTrident Medical Center, Charleston, SC, USA}

Abnormalities involving $c M Y C$ at $8 \mathrm{q} 24.1$ are characteristically observed in B-cell malignancies. Constitutive activation of $c M Y C$ typically occurs by juxtaposition of this oncogene with $I G H$ through a $t(8$ 14), which is the most common mechanism of oncogenesis in Burkitt lymphoma and to lesser extent in multiple myeloma. Herein we present a case of AML-M0 with an atypical $t(8 ; 14)$ which resulted in a $c M Y C$ $I G H$ fusion gene on the der(8), but with no reciprocal fusion on the $\operatorname{der}(14)$.

A 59-year-old African American woman was evaluated for anemia. She presented with abdominal pain, gastroesophageal reflux and gastritis. Her hemoglobin was low (7.7), white blood cell count elevated $(91,000 / \mathrm{ml})$ and peripheral blood smear was markedly abnormal $(70 \%$ blasts). The patient had a family history of various hematological conditions including leukemia, multiple myeloma and idiopathic thrombocytopenia purpura. She had no palpable splenomegaly. Peripheral blood flow cytometry showed the blasts were positive for multiple myeloid markers and negative for both non-specific and chloracetate esterases. Based on these studies, a diagnosis of AML-M0 was made. Chromosome analysis from blood showed all 20 cells had the clone 46, $\mathrm{XX}, \operatorname{del}(7)(\mathrm{q} 32 \mathrm{q} 34), \mathrm{t}(8 ; 14)(\mathrm{q} 24.1 ; \mathrm{q} 32)[20]$. Sequential fluorescence in situ hybridization demonstrated $c M Y C-I G H$ fusion on the der(8) and no fusion signal on the der(14). Interphase FISH with $c M Y C$ and $I G H$ probes showed $35 \%$ of nuclei with one fusion signal. Interphase FISH with $c M Y C$ break apart probe demonstrated $95 \%$ of nuclei with cMYC separation. FISH studies with ETO/AML1 [t(8;21)]; MLL (11q23) and
MYH11/CBFb[inv(16)] probes were all normal. The patient refused bone marrow biopsy and is responding poorly to induction therapy. Translocations resulting in cMYC-IGH fusion are typically associated with aggressive $\mathrm{B}$-cell malignancies. While it is not clear that the overexpression of cMYC is the primary abnormality in this case, the demonstration of $c M Y C-I G H$ fusion in AML-M0 is quite unusual and expands the hematological neoplasms associated with putative overexpression of $c M Y C$.

\section{1 \\ Two New Cases of Translocation Involving NUP98 in AML Patients \\ H. Bruyère ${ }^{a, b}$, D.E. Horsman ${ }^{b, c}$, K. Chipperfield ${ }^{a, b}$, D. Forrest ${ }^{b, d}$, M. Hudoba de Badyn ${ }^{a, b}$, K.W. Song ${ }^{b, d}$, R.K. Humphries ${ }^{b, e}$ \\ Department of Pathology and Laboratory Medicine, a ${ }^{a}$ ancouver General Hospital and 'British Columbia Cancer Agency, \\ ${ }^{\mathrm{d} T h e ~ L e u k e m i a / B o n e ~ M a r r o w ~ T r a n s p l a n t a t i o n ~ P r o g r a m ~ o f ~ B r i t i s h ~}$ Columbia, Division of Hematology, Vancouver General Hospital,

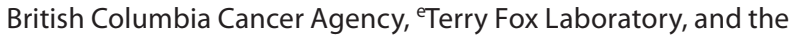 \\ bUniversity of British Columbia, Vancouver, BC, Canada}

Cytogenetic translocations play a major role in hematologic malignancies. They usually involve proto-oncogenes and disrupt the balance of cell growth, maturation and death. Gene partners in the translocation may be constant, as $A B L 1$ and $B C R$ in Chronic Myeloid Leukemia, or one gene may be recurrently involved, but with different partners, as MLL. The nucleoporin $98 \mathrm{kDa}$ (NUP98) gene is located at 11p15 and is implicated in translocations found in patients with myelodysplastic syndrome and acute leukemias. Although NUP98 translocations are rare, many rearrangement partners have been reported so far. We report two patients with prior breast cancer treated by chemotherapy who presented with acute myelogenous leukemia with a NUP98 translocation. A 59-year-old female was diagnosed with AML with monocytic differentiation, with a translocation $t(10 ; 11)(q 23 ; p 15)$. She achieved partial cytogenetic remission after induction chemotherapy (day 34) then completed cytogenetic remission after consolidation therapy (day 69). The patient received stem cell transplantation two months later and FISH at D100 showed 3/400 nuclei with two centromeric X signals and 391/400 cells with a centromeric X and a Yq12 signal. A 54-year-old female was diagnosed with a myelodysplastic syndrome with a translocation $\mathrm{t}(11 ; 20)(\mathrm{p} 15 ; \mathrm{q} 11.2)$ and clonal evolution. Two months later, transformation into AML happened and the $t(11 ; 20)$ was again detected in the bone marrow. The patient did not achieve morphologic remission. In both cases, FISH with BAC probes confirmed NUP98 involvement. Further studies are pending to identify the partner genes.

\section{2 \\ Segmental Jumping Translocations: A Review of a Rare Rearrangement Involved in Clonal Evolution and Disease Progression in Leukemias and Lymphomas}

P. Singh-Kahlon, C.F. Stephenson

Genzyme Genetics, Cytogenetic Labs, New York, NY, Phoenix, AZ, USA

Segmental jumping translocations are rare occurrences in hematopoietic malignancies. Nevertheless, a number of breakpoints on different chromosomes have been involved nonrandomly as 'donor sites' which have translocated onto several other 'recipient sites' on other chromosomes. The most commonly involved chromosomal segments appear to be 1q, 3q, 9q, 11q and 17q. The pathogenetic etiology of this 
mechanism has not yet been fully elucidated. Like double minutes (dmin), homogeneously staining regions (hsr) and amplifications, jumping translocations also serve as promoters of clonal evolution and disease progression in leukemias and lymphomas and, as such, are associated with poor prognosis.

The segmental jumping translocations usually result in several derivative chromosomes, reflecting the general instability of the 'donor sites' on chromosomes, but also result in deletions, duplications, rings and marker chromosomes. Consequently, this rearrangement results in multiploidy of the 'donor sites' segments involved (e.g., triploidy, tetraploidy, pentaploidy).

We present a review of cases with segmental jumping translocations involved in different hematopoietic malignancies from our laboratory's database. Possible pathogenetic and molecular mechanisms underlying the segmental jumping translocations will be discussed.

\section{3 \\ Frequency of 5' IGH Deletions in B-Cell Chronic Lymphocytic Leukemia/Small Lymphocytic Lymphoma (CLL/SLL)}

F. Nooraie, F. Quintero-Rivera, P.N. Rao

UCLA Clinical and Molecular Cytogenetics Laboratory, University of California at Los Angeles, Los Angeles, CA, USA

Although the involvement of the IGH rearrangements - mostly translocations - in B-cell chronic lymphocytic leukemia/small lymphocytic lymphoma (CLL/SLL) is common, this locus is not part of the routine FISH panel (13q14, 11q13, 17p13, 12 centromere) as provided by the vendors. In our study, conventional cytogenetics and FISH studies using the routine panel, plus the IGH 'break-apart' probe (Vysis, Inc) were successfully performed on 421 cases referred for CLL/SLL between Jan 2006 and Mar 2007. Based on the hematopathology and flow cytometry results, 289/421 (68\%) had a final diagnosis of CLL/SLL. The remaining was reported as normal (49), chronic lymphoproliferative disorder (36), different types of NHL (29), and other diagnoses (18) (from reactive changes to multiple myeloma).

Aberrant karyotypes were detected in 30\% (84/289), while FISH detected abnormalities in $70.5 \%(204 / 289)$ of the cases. 13q14 deletion was the most frequent anomaly seen in 64\% (161/289), followed by trisomy 12 in $19 \%$ (47/289), 11q deletion in $11 \%(28 / 289)$ and 17 p deletion in $6 \%(16 / 289)$, respectively. Adding the IGH probe to the FISH panel not only increased the diagnosis yield by $4 \%$, but ranked as the second most common abnormality in $31.8 \%(92 / 289)$, and the sole abnormality in $2.4 \%(7 / 289)$ of cases. Surprisingly, among the IGH abnormalities detected, translocations with unknown partners in the form of spilt signals $(1 \mathrm{R} 1 \mathrm{G} 1 \mathrm{~F})$ occurred in only a small group of patients $(21.7 \%$ or 20/92). However, deletion of 5' IGH (1F1R), corresponding to the variable gene segment $(I G H v)$ was the most recurrent aberration and was observed in $80.4 \%$ (75/92), considerably higher than previously reported (Fink et al., 2005). In 37\% (28/75) of these the only accompanied abnormality was $13 \mathrm{q}-$, which is known to be associated with a good prognosis in CLL. Aberrations associated with aggressive CLL such as deletions of $11 \mathrm{q}$ and $17 \mathrm{p}$ were concurrent with $5^{\prime}$ IGH deletion in only $6.6 \%(5 / 75)$ of cases.

Loss of DNA in the IGHv locus has been reported previously, though there remains considerable confusion about its biological and clinical significance in the cytogenetics community. Recent reports suggest that loss of DNA in this region is associated with other poor prognostic factors in B-CLL. However, Fink et al. did not observe a statistically significant correlation for IGHv deletions as detected by FISH and any of the prognostic factors (Rai stage, IGH $v$ mutation status, CD38 expn, other FISH anomalies). Since the sample size in the published studies were small $(n=50)$, we believe that our current study is a better representative of IGH aberrations in CLL/SLL, but more longitudinal studies are necessary to clarify if the IGHv deletion should be considered as an event of any clinical significance.

\section{4 \\ Chronic Lymphocytic Leukemia (CLL) Patients with High-Risk Cytogenetics Respond to Treatment with Flavopiridol: Study of 120 Patients (pts) with Relapsed, Genetically High Risk CLL}

\author{
N.A. Heerema, T.S. Lin, B. Fischer, G. Lozanski, K.A. Blum, \\ L.A. Andritsos, J.A. Jones, J.M. Flynn, M.E. Moran, S.M. Mitchell, \\ L.J. Schaaf, A.J. Johnson, L.L. Smith, A.J. Wagner, C.A. Raymond, \\ M. Phelps, J.T. Dalton, M.R. Grever, J.C. Byrd \\ The Ohio State University, Columbus, OH, USA
}

Background: Cytogenetic abnormalities predict response to treatment in CLL, with complex karyotypes ( $\geq 3$ unrelated abnormalities) by classical cytogenetics, and loss of TP53 (17p13.1) and ATM (11q22.3) by FISH being poor prognostic indicators following standard therapies (Byrd et al., JCO 24:437, 2006). We conducted Phase I and II studies of the drug flavopiridol, a cyclin dependent kinase inhibitor which induces p53-independent apoptosis in CLL cells in vitro, to determine whether pts with these high-risk cytogenetic features might respond to this drug.

Methods - Cytogenetics: Cytogenetics were assessed at the time of treatment initiation. Cells were stimulated with the B-cell mitogens pokeweed and phorbol 12-myristal 13-acetate and more recently also with CpG oligonucleotides for classical cytogenetics. A complex karyotype was defined as $\geq 3$ unrelated abnormalities. FISH included probes for TP53 and ATM to determine loss of those genes.

Treatment: Pts $(\mathrm{n}=120)$ with symptomatic, relapsed CLL $(\mathrm{n}=110)$ or small lymphocytic lymphoma $(\mathrm{n}=10)$ who failed (or could not receive) standard CLL therapy which included fludarabine, received flavopiridol by a pharmacokinetically (PK) determined schedule: $30 \mathrm{~min}$ intravenous bolus (IVB) followed by $4 \mathrm{~h}$ continuous IV infusion (CIVI) weekly for 4 doses every 6 weeks $(n=86)$ or 3 doses every 4 weeks $(n=$ 34) for up to 6 cycles. Three pts received $40 \mathrm{mg} / \mathrm{m}^{2} \mathrm{IVB}+40 \mathrm{mg} / \mathrm{m}^{2}$ CIVI, and all other pts received $30 \mathrm{mg} / \mathrm{m}^{2} \mathrm{IVB}+30 \mathrm{mg} / \mathrm{m}^{2} \mathrm{CIVI}$ for all doses $(n=28)$ or were dose escalated to $30 \mathrm{mg} / \mathrm{m}^{2} \mathrm{IVB}+50 \mathrm{mg} / \mathrm{m}^{2} \mathrm{CIVI}$ beginning with dose $5(n=14)$ or dose $2(n=75)$. Eight pts who experienced severe tumor lysis syndrome (TLS) did not undergo dose escalation as planned.

Results: Median age was 60 years (range, 36-84), 23 (19\%) were $\geq 70$ years of age, and $83(69 \%)$ were male. Median number of prior therapies was 4 (range, 1-14), and 119 pts had failed prior purine analog therapy. Pts had bulky Rai stage I/II $(n=27)$, III $(n=29)$ or IV $(n=64)$ disease; 88 (73\%) pts had bulky lymph nodes $\geq 5 \mathrm{~cm}$. Thirty-eight (32\%) pts had loss of TP53, 50 (42\%) had loss of ATM, and 54 (45\%) had complex karyotypes. Pts received a median of 3 cycles (range, $0.25-6$ ) of therapy, 24 completed all 6 cycles, and 6 continue to receive therapy. All 120 pts were evaluated for response, including 6 pts who received only 1 dose due to grade 4-5 tumor lysis syndrome or other complications. Fifty-six pts responded (47\%) by NCI Working Group criteria; 52 (43\%) with a partial response and 4 (3\%) with a complete response, including 1 pt with loss of TP53 and a complex karyotype. Responses were observed in 18 of 38 (47\%) pts with loss of TP53, in 28 of 50 (56\%) pts with loss of ATM and in 23 of $54(43 \%)$ pts with a complex karyotype. Overall, 43 of 89 (48\%) pts with high-risk cytogenetic features, and 42 of $88(48 \%)$ pts with bulky adenopathy responded to this new therapy.

Conclusions: Flavopiridol, given by this PK derived schedule, is clinically active in heavily pretreated, relapsed CLL pts with poor-risk cytogenetic features including complex karyotypes, loss of TP53 and loss of $A T M$, and in pts with bulky adenopathy. Cytogenetics is significant in determining treatment options for patients with CLL. 


\section{5 \\ Idiopathic Myelofibrosis with Ring Chromosome 10 Leading to Deletion of PTEN in a 21 Month Old Female}

J. Kelly ${ }^{a}$, A.M. Meloni-Ehrig ${ }^{a}$, J. Jahn ${ }^{a}$, J. Scheerle ${ }^{a}$, L. Matyakhina ${ }^{a}$ M. Motz ${ }^{a}$, N. Shahabazi ${ }^{a}$, J.Ramdas ${ }^{b}$, K. Zhang ${ }^{c}$, P.Dorion', J. Meck ${ }^{a}$, N. Christacos ${ }^{a}$, P.N. Mowrey ${ }^{a}$

aLaboratory of Cytogenetics, Quest Diagnostics Nichols Institute, Chantilly, VA, Departments of bediatric Hematology-Oncology, 'Pathology, Geisinger Medical Center Laboratories, Danville, PA, USA

The PTEN (phosphatase and tensin homologue) gene located at $10 \mathrm{q} 23$ is a tumor suppressor gene that is dysregulated in leukemia and other malignancies. This gene appears to act as a negative regulator, which plays a critical role in cell proliferation, survival, migration, and differentiation. In particular, authors observed that without functional PTEN, blood stem cells begin multiplying rapidly, showing diminished self-renewal capacity, and leading to the development of idiopathic myelofibrosis, and eventually leukemia with extramedullary involvement. In mouse models, it was reported that several days after PTEN depletion, stem cell numbers decreased in the bone marrow and increased in organs such as spleen and peripheral blood, with consequent stem cell death several days later [1,2]. To our knowledge, this is the first report of an idiopathic myelofibrosis with loss of PTEN in a human subject.

A 21 month old female presented with pancytopenia of uncertain duration. The physical examination revealed hepatosplenomegaly, spontaneous ecchymosis, and lymphadenopathy, which was considered reactive. All bacteriological tests were, in fact, negative. The peripheral blood showed a hemoglobin of $7.4 \mathrm{~g} / \mathrm{dl}$ and a WBC of 3.03 with 2 platelets and $83 \%$ lymphocytes. The initial bone marrow aspirate and biopsy showed no particles, and megakaryocytes, granulopoiesis, and erythropoiesis were markedly decreased. No abnormal blast population was identified by flow cytometry. Metabolic disorders such as Gaucher and Neimann Pick were ruled out by genetic work-up. JAK2 and ALPS panel were negative. Cytogenetic analysis of the initial bone marrow sample showed the loss of one copy of chromosome 10 and the presence of a ring chromosome in 12/20 (60\%) cells. Based on these cytogenetic findings, FISH analysis was performed with chromosome 10 centromere (D10Z1) and PTEN specific probes (Abbott Molecular/ Vysis). FISH was instrumental in confirming that the ring chromosome originated from chromosome 10 and had a deletion of the PTEN region. A follow-up bone marrow sample collected 2 days later showed decreased marrow cellularity. The overall diagnosis of this second sample was consistent with extensive myelofibrosis and persistent peripheral pancytopenia. Due to low platelets count and pancytopenia, she was transfused and later discharged. Cytogenetic analysis of this second sample showed a normal chromosome complement in all 20 cells analyzed. However, FISH detected one residual abnormal metaphase with the ring chromosome 10 and loss of PTEN. She continued to receive platelet transfusions every 3-4 days, but was not responsive to the transfusions. Bone marrow transplant was considered, however she did not have any HLA identical sibling. While waiting for an unrelated donor search, one month later, she was readmitted to the hospital but expired shortly after of intracranial hemorrhage with seizures and massive cerebral edema.

According to our findings, the disease progression in this patient appears to be similar to the aforementioned mouse model, in which loss of PTEN resulted in rapid proliferation and spontaneous migration of stem cells, with consequent myelofibrosis.

\section{References}

1 Moody et al: Anemia, thrombocytopenia, leukocytosis, extramedullary hematopoiesis, and impaired progenitor function in $\mathrm{Pten}^{+/-S H I P^{-/}}$mice: a novel model for myelodysplasia. Blood 15:4603-4610 (2004).

2 Zhang et al: PTEN maintains haematopoietic stem cells and acts in lineage choice and leukaemia prevention. Nature 441:518-522 (2006).

\section{6 \\ Black Tea (Camellia sinensis) as a Chemopreventive Agent in Oral Leukoplakia}

\author{
A. Halder ${ }^{a}$, R. Raychowdhury ${ }^{b}$, U. Das ${ }^{a}$, M. De ${ }^{a}$ \\ Departments of a Genetics and ${ }^{b}$ ENT Surgery, Vivekananda \\ Institute of Medical Sciences, Ramakrishna Mission Seva \\ Pratisthan, Kolkata, India
}

Oral carcinoma is the commonest malignancy found in adult Indian males, and the third commonest in adult Indian females. About half of all cases are found to be associated with precancerous lesions, chiefly leukoplakia. Eighty-two subjects with oral leukoplakia were administered black tea (Camellia sinensis) after undergoing micronuclei and chromosomal assays. The micronuclei assay was repeated at 6 months, and the chromosomal study at 1 year. The data of the first 15 patients at one year are presented; they show a significant decrease in the micronuclei frequency and chromosomal aberrations, which correlates with the clinical improvement observed. Several in vitro and animal studies have suggested the efficacy of tea in the chemoprevention of cancer. To the best knowledge of the authors this is the first work to specifically study the effect of black tea on oral leukoplakia.

\section{7 \\ The Evolutionary History of Mammalian Genome Organization \\ J. Wienberg \\ Institute for Anthropology and Human Genetics, Department \\ Biology II, Ludwig-Maximilians University, Munich, Germany}

The inspection of the morphology of chromosomes in different species has been a classical tool for evolutionary biologists for many decades. Molecular cytogenetic techniques introduced over the last 15 years provided various new insights into the mode and tempo of mammalian genome evolution. Molecular probes were helpful to distinguish mammalian chromosomes and chromosome segments on the basis of their DNA content rather than solely on morphological features such as banding patterns. The analysis of more than 100 mammalian genomes by chromosome painting showed that the gross organization of the genome of Eutheria (placental mammals) into chromosomes follows a simple basic architecture that, with some minor changes is almost completely conserved for more than 100 million years in various species of almost all extant mammalian orders. The main changes are movements and fission/fusion of large chromosome segments or of chromosome arms. Reciprocal translocations are the exception. The 'default' frequency of gross rearrangements would calculate less than two changes within 10 million years of mammalian evolution. Chromosome numbers may have changed significantly up and down in this fusion/fission process, but in most instances the main architecture still remains evident.

More detailed breakpoint analyses of chromosome rearrangements that occurred during evolution also gave some insights into the molecular changes in chromosome rearrangements. New array based and DNS sequencing tools have been used that now allow the analysis of chromosome breakpoint regions on the DNA level. In contrast to numerous chromosome rearrangements in cancer cells no new 'fusion genes' or dramatic 'position effects' of genes transferred to new sites have been documented. For example detailed breakpoint analysis of numerous translocations in a gibbon species revealed the presence of either segmental duplications or interspersed repeats at the very breakpoint locations. The progress in various molecular and molecularcytogenetic approaches including the current sequencing of various mammals of different taxa will have a significant impact on the further understanding of human genome evolution. 


\section{8 \\ Classification of Multiple Myeloma into Prognostic Groups Based on Karyotype and FISH}

H.A. Aviv, I. Maxwell, J. Wang, K. Vassallo, A. Ninan, C. Creese, D. Ang

Department of Pathology and Laboratory Medicine, Robert Wood Johnson Medical School and Robert Wood Johnson University Hospital, New Brunswick, NJ, USA

Multiple Myeloma (MM) can be classified according to gene expression profiles and cytogenetic results (EC for 'Expression/Cytogenetics') (Mahtouk et al., Clin Cancer Res 13:7289-7295, 2007). MM with Cyclin D1 overexpression includes the cytogenetic subgroups of trisomy $11 \mathrm{q}$ and hyperdiploidy (EC1.1), and $\mathrm{t}(11 ; 14)(\mathrm{EC} 1.2)$. MM with Cyclin D2 overexpression includes the Cytogenetic subgroups of $t(6 ; 14)$ and $\mathrm{t}(14 ; 16)$ and spiked MAF expression (EC2.1), and the $\mathrm{t}(4 ; 14)$ and spiked FGFR3 expression (EC2.2). The four groups have highly significant differences in event free survival, with patients in the EC2.2 group having the shortest survival, patients in the EC1.1 and EC2.1 groups having an intermediate survival and patients of the EC1.2 having the longest event free survival.

Our objective was to classify MM patients into these groups based on cytogenetics and FISH. In a pilot project, we collected cases ascertained by abnormal karyotypes and performed FISH analysis on 24 additional cases with normal karyotypes and at least $10 \%$ plasma cells in the bone marrow. We used centromeric probes for chromosomes 5 , 9 and 15 and the IGH break apart probe to distinguish hyperdiploid and $I G H$ translocation positive cases. Cases positive for $I G H$ rearrangement were probed for the CCNDI/IGH, IGH/MAF and IGH/FGFR3 fusions, and all abnormal cases were assessed for del(13q) with probes D13S319 and LAMP1 and for del(17p) with probes D17Z1 and p53. All probes were obtained from Vysis.

Sixteen cases had abnormal karyotypes and 6 additional cases had abnormalities on FISH. Thirteen cases were hyperdiploid, 5 had $t(11$; 14), 1 had $\mathrm{t}(14 ; 16)$, and no case had $\mathrm{t}(4 ; 14)$. Three cases had trisomy for only one chromosome and were excluded. Four of the hyperdiploid cases did not have trisomy 11q on karyotype, and the trisomy could not be established in the 2 hyperdiploid cases ascertained by FISH. Deletions of $13 q$ and $17 p$ were found in $38 \%$ and $23 \%$ of hyperdiploid cases. Two of the 5 cases with $t(11 ; 14)$ had trisomy 11q. Deletions of $13 q$ and deletion $17 p$ were found in $40 \%$ and $20 \%$ of cases with $t(11 ; 14)$. The $t(14$; 16) case had deletion of $13 q$ but not of $17 p$.

We conclude that all MM can be classified into one of the 4 prognostic groups by cytogenetics and FISH. Once a case has been classified, prognosis can be modified by the presence of trisomy 11q and deletions of $13 q$ and $17 p$. The role of trisomy 11q needs to be established in the hyperdiploid group, as not all hyperdiploid cases have trisomy 11q. A probe for trisomy 11q has to be included in the initial FISH panel. This pilot study allowed us to re-asses our procedures, and to devise a more informative and economic FISH screening for MM. Our subsequent results and work flow procedures in the analysis of samples from MM patients will be discussed.

\section{9 \\ Improved Detection of Chromosome Abnormalities in Patients with MDS Utilizing Interphase FISH}

\section{M.S. Matthews}

Acupath Laboratories, Plainview, NY, USA

Several testing modalities are employed in the diagnosis of myelodysplastic syndrome (MDS), including immunophenotyping, bone marrow biopsy analysis, fluorescence in situ hybridization (FISH) and conventional cytogenetic analysis (CCA). While each analysis provides important information in characterization of the disease, MDS remains a diagnostic challenge due to the inherent limitations of each test such as the inability to distinguish cell types, inter-observer variation, poor chromosome morphology and low mitotic index in the affected cell population.

CCA provides both diagnostic and prognostic information about MDS. While MDS patients with normal cytogenetic results have a favorable prognosis, these patients may benefit from further studies. Fluorescence in situ hybridization (FISH) in interphase nuclei of specific regions implicated in MDS permits rapid analysis of a larger number of cells than CCA.

In the current study, 30 individuals diagnosed with MDS, or suspected emerging MDS, and a normal result by CCA were selected for FISH analysis. In addition to FISH for detection of the most common chromosomal abnormalities associated with MDS ( -5 , del5q, -7 , del7q, +8 , and del20q), an expanded panel of probes for detection of less frequently detected abnormalities (del11q, del13q, 12p rearrangements, +21) was employed.

Of the specimens examined, 7/30 (23.3\%), were determined to have chromosomal abnormalities detected by FISH, not seen by CCA. Two of these (6.6\%) had a deletion of the long arm of chromosome 20 as the sole abnormality. Of the remaining 5 , one had deletion in the long arm of chromosome 7 , one had trisomy 8 , one had five copies of chromosome 8 , one had loss of the long arm of chromosome 11 and one had two abnormalities involving chromosomes 12 and 20 .

These findings demonstrate the ability of FISH to detect abnormalities not observed by CCA and indicate the utility of FISH as a useful adjunct in diagnosis of MDS compared to immunophenotyping, bone marrow biopsy analysis and CCA alone. Although the sample is small, the detection of one case with deletion of $11 \mathrm{q}$ reveals the value of the expanded FISH panel in detection of abnormalities in MDS.

\section{0 \\ Deletions on Derivative Chromosome 9 in CML with $t(9 ; 22)$ Arise Not Only when the Translocation Initially Occurs, but also as Part of the Clonal Evolution - Evidence from More than $\mathbf{5 0}$ Cases}

N.S. Mitter

Cytogenetics Laboratory, Dianon Systems (LabCorp), Stratford, CT, USA

It has been well established that CML/CNL/ALL/AML associated apparently balanced 'Philadelphia' chromosome rearrangement, $\mathrm{t}(9$; 22)(q34;q11.2) is often accompanied by deletion(s) $5^{\prime}$ of $A B L$ and $3^{\prime}$ of $B C R$ on the derivative chromosome 9 . The deletion breakpoints also vary among patients and, in some instances, span several megabases. These deletions are associated with a worse prognosis and the disease associated with a deletion is more refractile to treatment. The loss of arginosuccinate (ASS) gene probe signal is at a higher rate (45.5\%) in 'Philadelphia' chromosome $(\mathrm{Ph})$ positive patients with a variant translocation, compared to patients with a standard $\mathrm{Ph}$ translocation (17.6\%). Three pioneering studies, based on limited data, concluded that there is no clonal variation, i.e., all cells are either with or without ASS probe signal, implying that the $9 \mathrm{q}$ deletions occur at the time of the initial $\mathrm{Ph}$ translocation only.

Contrary to these conclusions, we have evidence from more than 50 cases in our database showing that the deletions on derivative chromosome 9 do become evident even during disease progression, or are coexistent in cells with other Ph-positive cells without deletions.

If the conclusions by Sinclair et al. (2000), Kolomeitz et al. (2001) and Huntly et al. (2001) are to be true, then it could also mean that in some patients both types of $\mathrm{Ph}$ translocations happen early during the genesis of disease, with one cell line type developing at a slower rate in the beginning (with the result of not being detected among 200 analyzed cells) and then having a selective advantage over the other cell line type. Based on the observations in as few as only 10 cells and up to 200 cells only in some instances, these studies seem to have limited information. Our analyses, based on a minimum of 200 cells for each patient, show that these deletions may also develop during disease pro- 
gression, and are associated with a genomic instability. Further, we show that the type of deletion (of only ASS gene, only $A B L$ gene or the whole complex, with no evidence for monosomy) can also vary among different cells from the same patient. Our results clearly illustrate the coexistence of both cell lines, with and without a deletion on the derivative chromosome 9 , in many patients with CML who have not even started taking Gleevac. The three-color probe set (Vysis) used in our analysis can differentiate between a loss of only $A S S$ region, only $A B L$ region and both $A S S$ and $A B L$ regions on the derivative chromosome 9. This method is superior to both the D-FISH and the three-color probe sets used by the three above-mentioned studies.

\section{1 \\ Unexpected Complexity of Supernumerary Marker Chromosomes Revealed by Microarray Comparative Genomic Hybridization}

K.D. Tsuchiya a , K. Opheim ${ }^{a}$, M. Hannibal ${ }^{b}$, A. Hing ${ }^{b}$, I.A. Glass ${ }^{b}$,

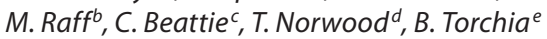

aDept of Laboratories, Children's Hospital and Regional Med Ctr, ${ }^{b}$ Dept of Pediatrics, Univ of Washington School of Medicine, 'Dept of Medical Genetics, Children's Hospital and Regional Med Ctr, ${ }^{\mathrm{d}}$ Dept of Pathology, University of Washington School of Medicine, Seattle, WA, eSignature Genomic Laboratories, LLC, Spokane, WA, USA

Supernumerary marker chromosomes (SMCs) are additional abnormal chromosomes that are unidentifiable by conventional cytogenetics and that vary considerably in size, structure, and chromosome composition. Characterization of the composition of SMCs can provide valuable information for genetic counseling, but even extensive FISH testing, including Spectral Karyotyping (SKY)/24-color FISH, can yield incomplete information. We have characterized a number of SMCs by microarray comparative genomic hybridization (array CGH) that were recently encountered in our clinical cytogenetics laboratory during the course of routine cytogenetic analysis of patients with multiple congenital anomalies. Array CGH not only correctly identified the chromosome of origin of all but one minute SMC, but it also defined specific regions of the chromosomes involved. The latter information would not have been obtained by SKY. In addition, array CGH uncovered unexpected complexity in the markers that may not have been appreciated even with extensive FISH analysis. These complexities included discontinuous regions of gain from the same chromosome, a marker composed of material from two different chromosomes, and a neocentric marker chromosome. In order to fully appreciate the composition of SMCs, our experience indicates that array CGH using an array that not only has extensive pericentromeric coverage, but also good overall genome representation is optimal.

\section{2}

\section{The Application of Interphase FISH Technique for Detecting Prenatally Common Chromosome Aneuploidies in Viet Nam}

H.M.V. Bui ${ }^{a}$, H.V. Bui ${ }^{a}$, T.N. Phung ${ }^{\text {b }}$, L.T. Mai ${ }^{b}$, T.D. Nguyen', T.C. Phan ${ }^{a}$

${ }^{a}$ Department of Histology, Embryology and Genetics, ${ }^{\text {'Tu Du's }}$ Hospital of Obstetric and Gynecology, 'Department of Obstetric and Gynecology, University of Medicine and Pharmacy,

Ho Chi Minh City, Viet Nam

Most aneuploidies diagnosed prenatally involve the autosomes 13 , 18,21 and sex chromosomes X and Y. The interphase fluorescence in situ hybridization (FISH) technique allows the rapid analysis of chromosome copy number in interphase cells. This technique has recently been applied in Viet Nam.
From January 2004 to December 2006, we applied the interphase FISH technique on 1628 pregnancies having a risk of chromosome aneuploidy. Standard cytogenetic analysis of cultured amniotic fluid cells was performed when abnormal interphase FISH results were obtained, failure of the interphase FISH technique, for pregnancies at risk for a balanced translocation, and when requested by the patient. Confirmation and follow up of all normal FISH results was obtained by contacting the patient following the birth of the baby. Aneuploidies detected in $151(9.29 \%)$ samples included trisomy 21 , trisomy 18, trisomy 13, monosomy X, XXY, XXX and triploidy. There were no false negative or false positive results.

We conclude that the interphase FISH technique is both a fast and accurate method for routine prenatal diagnosis of the common aneuploidies in high-risk pregnancies.

\section{3 \\ Validation of Fluorescence in situ Hybridization Assays: BETAINV versus Mean plus 3 Standard Deviations for Calculation of Normal Cut-Off Values \\ L.R. Rowe ${ }^{a}$, L.S. Rector ${ }^{a}$, A.N. Lamb ${ }^{a-c}$, A.R. Brothman ${ }^{a-d}$, S.T. South ${ }^{a, c}$ \\ anstitute for Clinical and Experimental Pathology, Associated Regional and University Pathologists (ARUP) Laboratories, \\ ${ }^{b}$ Department of Pathology, 'Division of Medical Genetics, Department of Pediatrics, 'Department of Human Genetics, University of Utah, Salt Lake City, UT, USA}

Laboratories must establish the performance characteristics of assays that modify an FDA-cleared or approved test, or use a test in which the manufacturer does not provide test characteristics. The vast majority of DNA probes used in fluorescence in situ hybridization (FISH) testing of cytogenetic samples are not FDA-approved. As a result, the American College of Medical Genetics (ACMG) and the Association for Molecular Pathology (AMP) have issued guidelines for FISH test development and implementation, including methods to establish reportable reference ranges. In 2005, the ARUP Cytogenetics laboratory began validating FISH probes for use in interphase cells using a standardized validation protocol. The signal patterns identified in 500 cells from each of 20 normal samples were recorded to establish reference range cutoff values, using a statistical calculation of the mean plus 3 standard deviations (mean $+3 \mathrm{SD}$ ). Recently however, the ACMG and AMP have suggested that the Microsoft Excel beta inverse (BETAINV) function is a more appropriate statistical approach for determining normal cutoff values and that cutoff values should be established for different numbers of cell counted. Consequently, we compared the differences between cutoff values established using the mean $+3 \mathrm{SD}$ and BETAINV methods for $100,200,300,400$, and 500 cell counts. The BETAINV method produced consistently higher normal cutoff values compared to the mean $+3 \mathrm{SD}$ method, with an average increased normal reference range of $0.9 \%$ (range 0.4 to $2.1 \%$ ) in 500 cell counts. We observed the greatest difference in cutoff values generated by the two methods in cell counts of 200 and less. This suggests that increasing the cell count from 200 to 500 is useful for detection of suspected lowlevel mosaicism, but may not be necessary when a sample is clearly abnormal after 200 cells are scored. Using BETAINV, a trend toward lower cutoff values was observed as cell counts increased, confirming the relationship between increased sensitivity and higher numbers of cells scored. As a result of this study, we have adopted the BETAINV method to determine normal reference range cutoffs.
Preprint Cytogenet Genome Res 121/1/2008

Abstracts will be available online,

free of charge 


\section{4 \\ Assessment and Validation of the BioView Duet System for Automated Scoring of UroVysion}

D.J. Wolff, R. Rogers, D.I. Quigley

Department Pathology and Laboratory Medicine, Medical University of South Carolina, Charleston, SC, USA

The BioView Duet system integrates an automated scanning microscope and an image analysis system that is FDA approved for scoring the Abbott/Vysis UroVysion probe set. For initial validation studies, 82 samples were manually read and subsequently assessed on the BioView system and results were compared. The overall concordance rate was $90 \%$ (16 cases were positive and 58 cases were negative by both methods). Five cases (6\%) were positive by BioView and negative by the manual method while 3 cases (4\%) were positive by the manual method and negative by BioView. Of these one had significant fluorescence fading and 2 had low levels of positive cells ( 1 in 124 cells and 1 in 130 cells). Technologist workload recording for reading the BioView images was significantly less than for manual slide scoring (average $20 \mathrm{~min} / \mathrm{slide}$ for BioView and $32 \mathrm{~min}$ for manual). Based on the advantages of the BioView system including decreased technologist time, improved workflow with better turn-around times and increased reimbursement, the BioView system was integrated as the clinical UroVysion scoring method. Quality control monitoring of the first 230 cases scored by the BioView System compared to 234 cases that were scored using the manual method revealed positive rates of $15 \%$ and $20 \%$ for BioView and the manual method, respectively. Insufficient sample rates for BioView and manual were $6.5 \%$ and $6.4 \%$, respectively. Several changes to the BioView reading/scoring have been instituted and our experience is that this automated system provides a sensitive and cost-effective alternative to manual scoring.

\section{5 \\ A Statistical Method for Calculating FISH Reference Ranges over a Large Number of Values}

\section{A.L. Ciolino, M.E. Tang, R.J. Bryant}

Department of Pathology and Laboratory Medicine, Fletcher Allen Health Care, Burlington, VT, USA

Fluorescent in situ hybridization (FISH) has become an essential tool for diagnosing and monitoring hematological disease. Testing for minimal residual disease requires precise and accurate normal cutoffs. Establishing appropriate cut-offs for FISH is complex because the values fall in a binomial distribution rather than a Gaussian distribution and the mathematics are unfamiliar to most laboratorians. We describe the theory of the binomial distribution as applied to FISH and give a detailed example of how to create a Microsoft Excel spreadsheet that will correctly calculate the cut-off limit for any number of nuclei counted. We discuss the importance of the initial evaluation and the limitations to the assay's sensitivity imposed by the number of nuclei scored in the initial probe evaluation.

\section{6}

Prenatal Diagnosis of a de novo Unbalanced X-Autosome Translocation by Array-CGH in a Female Fetus with an Increased Nuchal Translucency

E. Kolomietz ${ }^{a}$, J. Mazurkiewicz ${ }^{a}$, D. Chitayat ${ }^{b}$

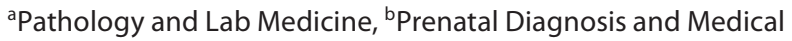
Genetics Program, Mount Sinai Hospital, University of Toronto, Ont., Canada

Increased nuchal translucency (NT) in the first trimester of pregnancy has now been clearly identified as a marker for aneuploidies. We report a prenatal case with microarray comparative genomic hybridization characterization of an abnormal derivative chromosome $\mathrm{X}$ identified through increased NT. Amniocentesis was performed because of an increased NT and revealed an abnormal de novo karyotype: $46, \mathrm{X}$, add $(\mathrm{X})(\mathrm{q} 27)$. To characterize the origin of the additional material on the long arm of chromosome $\mathrm{X}$, we performed an aCGH analysis using CytoChip (BlueGnome Ltd., Cambridge, UK) microarray providing an average of $0.5 \mathrm{Mb}$ resolution. aCGH revealed a deletion of the distal portion of the long arm of chromosome $\mathrm{X}$ and a duplication of the terminal portion of the short arm of chromosome 18. Therefore, the fetus carries a derivative chromosome $\mathrm{X}$ arising from a de novo unbalanced translocation: $\operatorname{der}(\mathrm{X}) \mathrm{t}(\mathrm{X} ; 18)(\mathrm{q} 28 ; \mathrm{p} 11.31)$. aCGH based breakpoint mapping allowed precise characterization of segmental aneuploidy. The deleted Xq28 region includes the region containing the gene MECP2. Deletion of this gene is found in females with Rett syndrome. aCGH analysis is a powerful tool to identify small de novo unbalanced chromosomal abnormalities and can be successfully applied in prenatal diagnosis.

\section{7 \\ Evaluation of Anencephaly, Rachischisis, and Omphalocele in a Stillborn Cynomolgus Monkey (Macaca fascicularis)

\author{
S.M. Gardner ${ }^{a, b}$, C.M. Moore ${ }^{a, e, f}$, E.J. Dick, G.B. Hubbard ${ }^{f}$ \\ B.G. Dunn ${ }^{b}$, V. Williams ${ }^{a}$, C. Keller ${ }^{a, c}$, M.D. Davis ${ }^{d}$
} \\ Departments of a Cellular and Structural Biology and ${ }^{\mathrm{b} C l i n i c a l}$ Laboratory Sciences, 'Greehey Children's Cancer Research Institute, ${ }^{d}$ Research Imaging Center, University of Texas Health Science Center at San Antonio; 'Department of Genetics and fSouthwest National Primate Research Center, Southwest Foundation for Biomedical Research, San Antonio, TX, USA}

A stillborn cynomolgus monkey (Macaca fascicularis) with multiple congenital anomalies was found in one of the breeding cages at the Southwest Foundation for Biomedical Research (SFBR) in San Antonio, Texas. The tissues were in an advanced state of decomposition. Physical findings include low birth weight, anencephaly, rachischisis, facial abnormalities, omphalocele, and malrotation of the gut with areas of atresia and intussusception. A patent ductus arteriosus and patent foramen ovale were also observed. The macaque had normal male external genitalia, but undescended testes. Gestational age was not known but was estimated from femur length and other developmental criteria. Cultures were established, but no growth was obtained. DNA extraction from liver and muscle produced degraded DNA samples. Further evaluation of the animal included extensive imaging of the skeletal and neural tissue defects and an attempt to evaluate the cytogenetic status of the animal through hybridization with human DNA enumeration probes. A discussion of the developmental sequelae of severe neural tube defects and schisis association will be presented.

\section{8 \\ Update on Placental Mesenchymal Dysplasia, Chimeras, Mosaics and P57 ${ }^{\mathrm{KIP2}}$ \\ U. Surti, L. Hoffner \\ Magee-Womens Hospital and Magee Womens Research Institute, University of Pittsburgh, Pittsburgh, PA, USA \\ Placental mesenchymal dysplasia (PMD) has been a diagnostic problem. Ultrasound findings show an enlarged partially cystic pla- centa resembling a partial hydatidiform mole (PHM) with a fetus. His- tologically PMD lacks trophoblastic abnormalities associated with PHM. Fetal outcome of PMD is variable and unpredictable. About 60 cases of placental mesenchymal dysplasia have been reported in the literature, but the genetic origin of only a few cases has been deter- mined including multiple cases with androgenetic/biparental mosa-}


icism or chimerism. In addition to morphological, histological, and genetic testing, immunostaining using a maternally expressed $\mathrm{p} 57^{\mathrm{KIP} 2}$ antibody has proved useful in detecting androgenetic cell populations. The $\mathrm{p} 57^{\mathrm{KI} 2}$ gene is paternally imprinted and maternally expressed; therefore, the positive staining of its protein indicates the presence of a functional maternal allele. Because androgenetic cells lack a maternal genome, p57 $7^{\mathrm{KIP} 2}$ immunostaining is absent.

Simultaneous $\mathrm{p} 57^{\mathrm{KIP} 2}$ immunostaining and FISH using FICTION technique has allowed us to elucidate the cytogenetic identity and distribution of multiple cell populations in an individual case. We report on 65 cases of hydropic placentas, correlating cytogenetic and p57 KIP2 immunostaining results. Additionally, cases with unusual p57 ${ }^{\mathrm{KIP} 2}$ staining patterns are discussed. Also included are one case of androgenetic non mosaic trisomy, 4 mosaic conceptions (3 diploid/triploid and one diploid/tetraploid; 6 chimeric/mosaic conceptions with androgenetic/biparental cell lines; and 2 cases of placental mesenchymal dysplasia (PMD). Our cases along with the review of current literature provide insight into novel mechanisms of origin for mosaic and chimeric conceptions.

\section{9 \\ No Association of Skewed X Inactivation with Recurrent Abortion or Trisomy

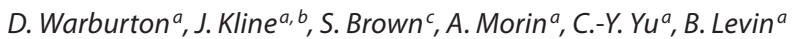 \\ ${ }^{\mathrm{a} C o l u m b i a}$ University and ${ }^{\mathrm{b} N e w}$ York State Psychiatric Institute, New York, NY, CUniversity of Vermont, Burlington, VT, USA}

Previous studies suggested an association between highly skewed $\mathrm{X}$ inactivation (HSXI) and recurrent spontaneous abortion. We hypothesized that this might reflect an association between HSXI and trisomy, due to $\mathrm{X}$ chromosome rearrangements or mutations that could induce both skewing and premature ovarian aging through oocyte atresia. Alternatively, an anomalous X chromosome could cause embryonic death in male conceptions. To test these hypotheses we measured XCI skewing in 324 women ascertained through a karyotyped spontaneous abortion and 427 age-matched controls of women with livebirths at the same hospital. Skewing was measured by the HUMARA assay with control Rsal and MIC2 digestion and ratios were calculated to lie between 50 and $100 \%$. For a sub-sample of specimens, ratios were compared with those obtained in a commercial laboratory, giving an ICC value of $>90$. Samples repeated in our own laboratory using the same PCR products had an ICC of $>95$. Because skewing $\geq 90 \%$ was infrequent $(\sim 2 \%)$ in our sample, we defined HSXI as $\geq 85 \%$.

A skewing ratio $\geq 85 \%$ was found in $5.4 \%$ of controls $(n=427), 5.5 \%$ of women with trisomic losses $(n=163)$ and $2.2 \%$ of women with normal male losses $(n=46)$. The odds ratio for highly skewed vs. nonskewed was 1.2 (95\% CI 0.5-2.8) among women with trisomic losses and 0.3 (95\% CI 0.04-2.6) among women with male losses. Thus our study does not support a relationship between HSXI and either trisomic or normal male losses. HSXI was also found in only $3.6 \%$ of women $(n=56)$ with recurrent losses. In secondary analyses, the distribution of skewing was significantly different among non-trisomic abnormal losses $(\mathrm{p}<0.02)$ and the odds ratio for HSXI was $2.1(95 \% \mathrm{CI}$ $0.8-5.6)$. The association appeared to be due to increased HSXI (21.1\%) among cases with monosomy X losses $(n=19)$. Classification by trisomy type also raised the possibility that HSXI was increased (13.2\%) among non-acrocentric trisomies other than $16(\mathrm{p}<0.04)$. Because of small samples and the multiple tests performed, these results require confirmation. Notably, Bretherick et al. (2003) found that HSXI was highest among women with similar trisomies. All 40 women with HSXI had normal conventional karyotypes, and high-resolution aCGH on 15 of these revealed no significant X chromosome changes. We also measured skewing from left and right buccal smears of all 40 women. Correlation between skewing ratios in blood and buccal smear samples was modest $(r=0.7)$. Among 42 women with HSXI in blood, only 3 had HSXI in both left and right buccal smears. Thus the skewing ratio is unlikely to be due to primary selection, but more likely to be the result of random selection among small stem cell populations or secondary selection which can differ among tissues and changes with age.

\section{0 \\ Sex Chromosome Loss in Lymphocyte Culture: Incidental or Clinically Relevant Finding; When Do You Report It?}

\section{S. Shetty, J.E. Chernos}

Alberta Children's Hospital, Faculty of Medicine, Department of Medical Genetics, University of Calgary and Calgary Health Region, Calgary, Alta., Canada

Sex chromosome aneuploidy is detected occasionally in some metaphases of stimulated lymphocyte cultures from adult patients presenting with reproductive issues (recurrent miscarriages, infertility etc) or abnormal gonadal function. In a cytogenetic laboratory, the observation of low level mosaicism for sex chromosome loss or gain particularly in the context of reproductive concerns can be difficult to differentiate between clinically relevant or in vitro artefacts. In the literature, there are very few published data available for interpreting these findings. In absence of recent information regarding loss or gain of sex chromosomes in these patients, we undertook a prospective study. Cytogenetic analysis was performed to assess X or Y chromosome aneuploidy in an effort to determine the clinical significance of loss or gain of sex chromosomes in more than one cell in lymphocyte cultures. A total of 17,512 cells from 576 patients aged 20 and older (irrespective of the indication for analysis) were screened cytogenetically. Patients under 20 years of age and patients over 20 years with a constitutional chromosomal abnormality identified were excluded. Using statistical analyses, comparison between the groups with and without reproductive issues or suspected sex chromosome anomalies and age will be performed to determine the significance. Males referred for repeat pregnancy loss in the partners or infertility will be used as controls for loss of the X chromosome. To determine the effect of age on sex chromosome aneuploidy, the population will be stratified into 20-49 and $50+$. Autosomal aneuploidies will be considered as a control for random losses in culture and compared with sex chromosome aneuploidies. Preliminary analysis of the data will be used to determine the statistical significance of low level mosaicism due to loss or gain of sex chromosomes and presented to provide guidance for interpreting significance of this finding in different age groups as well as clinical indications.

\section{1 \\ Cytogenetic Study of 245 Infertile Men in Taiwan: Secret behind the Number?}

M.H. Cheng ${ }^{a, c, d}$, P.H. Wang a, c, M.J. Yang ${ }^{a, c}$, H.T. Chao ${ }^{a, c}$, S.P.Chang ${ }^{a, c}$, K.C.Chao ${ }^{a, c}$, J.S. Huang ${ }^{b, c}$

Departments of a Obstetrics and Gynecology and ${ }^{b}$ Urology, Taipei Veterans General Hospital, 'National Yang-Ming University Schools of Medicine, Taipei, Taiwan, ${ }^{\mathrm{d}}$ Department of Laboratory

Medicine, School of Medicine, University of California,

San Francisco, CA, USA

Chromosomal aberration plays a major role in male infertility. The reported percentage of infertile males with chromosome abnormalities varies in different populations, ranging from 2.2 to $14.3 \%$. In Taiwan, estimated $15 \%$ of all couples suffer from reproductive difficulty, and one-third of them are attributed to male factors. However, the chromosomal complements in male infertility have not been well studied. We reported here cytogenetic analysis of 245 unselected males who visited the Taipei Veterans General Hospital during January 1998 to December 2007 due to reproductive failure. 
Approximately $24.1 \%$ (59/245) individuals in this particular group showed chromosomal abnormalities. The most frequent sex chromosome abnormalities are $47, \mathrm{XXY}$ (37 cases, $15.5 \%$ ), followed by mosaic sex chromosome abnormalities (11 cases, $4.5 \%)$, XX male ( 2 cases, $0.8 \%$ ), sex chromosome-autosome translocation (1 case, $0.4 \%$ ), and $\mathrm{Y}$ chromosome pericentric inversion ( 1 case, $0.4 \%)$. The autosomal abnormalities are mainly balanced translocations (5 cases, $2.0 \%)$. It is noteworthy that the frequency of chromosomal aberrations, in particular non-mosaic and mosaic XXY, in our patients is higher than that in most studied male patient populations. The findings imply a relatively unique cytogenetic epidemiology for male infertility in Taiwan.

Klinefelter syndrome is a well-known genetic disorder, but it was diagnosed in our patients only when cytogenetic test was ordered due to infertility at a relatively late age (an average of 34.2 years old). This suggests that this disorder appears to be severely overlooked. Delayed and/or under diagnosis of Klinefelter syndrome and other chromosomal disorders would result in unnecessary psychological burden on the patients and their families, which otherwise would be prevented or better managed. Our findings provide a foundation for developing appropriate strategies to improve reproductive health and related social issues in Taiwan, such as counseling and better clinical management of the patients.

\section{2 \\ Moving Cytogenetics from Bands into Bioinformatics \\ C. Lese Martin}

Department of Human Genetics, Emory University, Atlanta, GA, USA

With the rapid integration of genome-wide array-based copy number analyses into clinical cytogenetics testing, cytogeneticists are faced with developing new skill sets for interpreting 'Molecular Karyotype' results and determining their clinical relevance. While G-banding analysis involves a subjective estimate of the chromosomal bands involved in a particular aberration, array analysis offers an objective method for breakpoint determination based on genome sequence coordinates. However, just as with G-banding, chromosomal imbalances need to be categorized as either pathogenic and thus, clinically relevant or benign variants that are most likely not related to the abnormal clinical presentation. In order to accurately distinguish between these categories for Molecular Karyotype results, it is necessary to utilize multiple genomic and bioinformatics resources to provide an appropriate clinical interpretation. This workshop will provide an overview of the various web-based databases available for array analysis and review examples of results from clinical cases utilizing these resources.

\section{3 \\ Utilization and Initial Implementation of a Whole Genome SNP Array for Clinical Analysis: Advantages, Problems and Unexpected Findings}

\section{S. Schwartz, C. Tan, L. Henderson, R. Nicolae, C. Ober, D. Waggoner, A. Murmann}

Department of Human Genetics, University of Chicago, IL, USA

Over the past 15 years there has been a revolution in the detection of chromosomal abnormalities, first by the use of FISH for a directed examination of changes involving specific segments of DNA, to array technology in which changes in the entire genome can be delineated in one experiment. This latter technology has been especially useful in the detection of small duplications and deletions and the elucidation of new subtle deletions. However, there is still much debate regarding the methodology that should be used in the routine clinical study of patients. In this study, we present data from a beginning evaluation of a whole genome 250k SNP array to determine how effective this technol- ogy would be in detecting abnormalities and the complexity of the work involved to complete this analysis. To accomplish these goals we have utilized a whole genome analysis to study 213 different specimens. These samples have included four different types of specimens including chromosomally abnormal cases that had initially been detected through routine chromosomal studies (169 duplications or deletions); microdeletion cases that could not be detected with routine chromosome studies but were delineated with FISH or molecular analysis (18 microdeletions); individuals with phenotypic abnormalities, but were not found to have a chromosomal abnormality (18 individuals); and individuals who neither had a chromosomal or phenotypic abnormality (11 individuals).

The analysis and interpretation of our raw data was accomplished by utilizing two different algorithms that employed two different control populations. While most of the abnormalities detected in this study could be elucidated utilizing either of these analytic algorithms, they appeared to be somewhat complimentary and were seen to increase the overall detection of abnormalities. Overall the findings from our studies have shown that this methodology has been very effective in detecting chromosomal abnormalities and have provided a number of unexpected findings. When utilizing the array analysis to study patients that have been previously studied by chromosomal analysis an additional 50 true abnormalities (both deletions and duplications), were detected. These were confirmed by FISH studies, and seen in both patients that were not initially found to have an abnormality, as well as those with other abnormalities. While some of these were large, some of the abnormalities were as small as $40 \mathrm{~kb}$. The array analysis additionally clarified some of the abnormalities, as well as detailing some complexity not initially seen. In addition, some cases initially thought to be abnormal were shown to actually be normal. A number of subtle microdeletions that could not be seen with routine analysis as small as 200 $\mathrm{kb}$ were detected by the array studies. In approximately $22 \%$ of the phenotypically abnormal but previously chromosomally normal individuals, an abnormality was detected. Additionally, using both algorithms for our analysis, an increased number of benign copy number variations (including over $50 \mathrm{CNVs}$ not previously reported) were detected. In order to implement this analysis clinically, we have utilized a stepwise approach, including determining if genes are associated with the detected deletions and duplications and if these abnormalities were familiar. Based on this we believe that we can use this technology to detect abnormalities that are $300 \mathrm{~kb}$ or greater, although smaller deletions have already been detected in some of our cases.

\section{4}

\section{The Fine-Scale and Complex Architecture of 1086 Human Copy Number Variant (CNV) Regions as Defined by Custom High-Density Oligonucleotide Microarrays}

\section{Lee ${ }^{a, b}$, G.H. Perry ${ }^{a, c}$, A. Ben-Dor ${ }^{d}$, A. Tsalenko ${ }^{d}$, N. Sampas ${ }^{d}$, L. Rodriguez-Revenga $a^{a, b}$, C.W. Tran ${ }^{a}$, A. Scheffer ${ }^{d}$, I. Steinfeld ${ }^{d}$, P. Tsang ${ }^{d}$, N.A. Yamada ${ }^{d}$, HS. Park ${ }^{e}$, J.-I. Kim ${ }^{e}$, J.-S. Seo ${ }^{e}$, Z. Yakhini ${ }^{d}$, S. Laderman ${ }^{d}$, L. Bruhn ${ }^{d}$}

aDepartment of Pathology, Brigham and Women's Hospital, Boston, MA, 'b Harvard Medical School, Boston, MA, 'School of Human Evolution and Social Change, Arizona State University, Tempe, AZ, ${ }^{d}$ Agilent Technologies, Santa Clara, CA, USA,

eDepartment of Biochemistry, College of Medicine, Seoul National University, Seoul, South Korea

Copy Number Variants (CNVs) are highly prevalent in the human genome and may play an integral role in normal human phenotypic variation and disease susceptibility. Initial screens have used a variety of technologies to identify thousands of human $\mathrm{CNV}$ regions that are now being systematically catalogued and utilized in clinical interpretations of genomic tests. Unfortunately, most of these studies have an appreciable false positive rate and also provide limited resolution of the actual size and structure of these CNV regions. To address this limited understanding, we have constructed a custom oligonucleotide array 
containing 470,143 distinct 60mer probes, selected with an approximate $1 \mathrm{~kb}$ spacing within and flanking 2,191 common human CNV regions annotated in the Database of Genomic Variants. We have reinterrogated the genomic DNAs from 30 HapMap individuals and observed copy number differences at 1,086 of the 2,191 targeted CNV regions. Our results revealed that 1,020 of these CNV loci were actually smaller in size than what is recorded in the Database of Genomic Variants (DGV), based on previously published studies. A reduction in size of more than $50 \%$ was observed for $876 \mathrm{CNV}$ regions, consistent with the notion that the total genomic content of currently known common human CNVs is likely smaller than previously thought. In addition, approximately $8 \%$ of the CNV regions observed in multiple individuals exhibited genomic architectural complexity in the form of smaller CNVs within larger ones and CNVs with inter-individual variation in breakpoints. This information has been deposited into DGV for immediate use in array CGH-based clinical cytogenetic diagnoses. Moreover, future association studies that aim to capture the potential influences of CNVs in disease phenotypes will need to consider how best to account complex CNVs.

\section{5 \\ Utility of a BAC Emulated Oligonucleotide Array CGH in a Clinical Setting}

A. Patel, S.H.L. Kang, C.A. Shaw, T. Sahoo, S. Lalani, C.A. Bacino,

P. Stankiewicz, C.A. Shaw, A.C. Chinault, A.L. Beaudet, J.R. Lupski, S. Neill, A.N. Pursley, P.A. Ward, S.W. Cheung

Baylor College of Medicine, Department of Molecular and Human Genetics, Houston, TX, USA

Array comparative genomic hybridization (array-CGH) allows genome-wide screening for copy number changes that is limited only by the probes that are present on the array. Recently the trend has been toward the use of commercially manufactured high-density oligonucleotide microarrays to detect these changes. However, major hurdles in transitioning this technology to the clinic include the need to establish a reliable and cost-effective method to 1) confirm copy number changes, and 2) determine whether the observed change is a normal variant or pathological. To overcome this, we have developed and validated an oligo-based BAC emulation array using the Agilent platform for use in clinical practice. This array contains $\sim 44,000$ oligos grouped into the same BAC sequences that have been previously used on our array-CGH assay. The microarray was developed by initially selecting 105,000 oligos covering the regions of interest and using empirical hybridization data and oligo distribution analysis to select an optimized $44 \mathrm{~K}$ oligo array. Therefore, this $44 \mathrm{~K}$ oligo array remains primarily focused on known disease regions and telomeres combined with backbone coverage of the entire genome and emulates the BAC arrays with which we have generated a valuable database with results from over 8000 patients. Using this array we have analyzed to date 3912 postnatal cases. Overall, a copy number gain or loss in a genomic region was detected in 27\% (1058/3912) of the cases. Of these, 435 (41\%) cases had an imbalance in a known clinically significant region and in 519 (49\%) cases, parental FISH or CMA studies were needed to determine if the copy number change was a familial copy number variant or a clinically significant de novo event. Parental samples were received for 165 cases and $93 \%$ of these were classified as familial copy number variants and $7 \%$ were classified as de novo events. Known or common CNVs were detected in $3 \%$ of the cases. Comparing our experience of BAC arrays to the OLIGO arrays we found that the OLIGO arrays are more reliable and more sensitive/robust (up to 2-fold) than the BAC arrays because of improved dynamic range. The increased reliability of the OLIGO data negates the need for parallel dye-swap experiments. The increased sensitivity of the OLIGO data as well as advances in the software used to calculate and display copy number changes permits more accurate determination of smaller genomic imbalances that are not statistically significant on the BAC versions.

\section{6}

Genome-wide and Targeted Analysis of Chromosomal Abnormalities Using High Density Oligonucleotide Microarrays

V.L. Ott, R.R. Selzer, J. Geoghegan, P.S. Eis, T.A. Richmond, J. Kitzman, J. Norton, R.D. Green

Roche NimbleGen, Madison, WI, USA

Microarray-based Comparative Genomic Hybridization (array $\mathrm{CGH}$ ) methods have been widely used to investigate genome-wide chromosomal abnormalities associated with cancer and developmental disorders. Historically, microarray-based assessment of copy number changes has been performed with BAC array CGH, which is limited to $0.1-1 \mathrm{Mb}$ resolution. Recently, oligonucleotide-based array CGH has been adopted, providing orders of magnitude increase in resolution as compared with BAC array CGH and conventional chromosome analysis. Not surprisingly, the application of array CGH to genetics research has enabled the discovery and fine characterization of novel genomic disorders including 17q21 (Sharp, 2006), 15q24 (Sharp, 2007), 1q41q42 (Shaffer, 2007), and 16p11.2-p12.2 (Ballif, 2007) microdeletion syndromes.

We have developed the first array CGH platform containing 2.1 million long oligonucleotide probes per array, offering a probe every $1 \mathrm{~kb}$ across the entire human genome in an unbiased tiling-path design format. The 2.1 M feature human whole-genome array enables detection of deletions and amplifications down to $\sim 5 \mathrm{~kb}$ in size, as well as breakpoint mapping of larger sized chromosomal changes at the gene level. In some cases, chromosomal abnormalities previously characterized on lower resolution platforms show distinct breakpoints as well as complex structural variation (e.g. a deletion immediately adjacent to an amplification) when examined with higher probe density. In addition to whole-genome arrays, we have developed higher throughput multiplex formats of both our $385 \mathrm{~K}$ and $2.1 \mathrm{M}$ feature microarrays (e.g., 4 sub-arrays of $72 \mathrm{~K}$ probes each on a single slide) for targeted analysis of single genes or regions, or multiple loci of interest in the genome. These new microarray technology improvements will enable rapid progress in the detection and characterization of chromosomal abnormalities associated with genomic disorders, cancer, and other complex diseases.

\section{7 \\ Validation of Whole Genome Microarray SNP Testing}

\section{J.H. Tepperberg, B. Williford, P.R. Papenhausen}

Laboratory Corporation of America, Research Triangle Park, NC, USA

High resolution whole genome chromosome microarray technology allows the detection of subtle genomic imbalances in patients with idiopathic MR and developmental delay. Fifty-three patients with known genetic imbalances and 7 patients who were normal in previous CGH BAC array, FISH or chromosomes testing, were analyzed with the NSP portion of the $500 \mathrm{~K}(260,000$ SNPs) SNP array from Affymetrix. Fifty-one cases were confirmed positive by a secondary method and 7 were normal by both methods. Fifteen of the 51 true positive cases were below $500 \mathrm{~kb}$ or were in a region with greater than $50.0 \%$ known copy number variants. Overall concordance was $96.6 \%$ with sensitivity at $96.2 \%$ and specificity of $100.0 \%$. DNA copy number changes ranged in size from $98 \mathrm{~kb}$ to $4.8 \mathrm{Mb}$. Four cases were positive with the Affymetrix array and negative by other methods. These four cases appear to represent true copy number changes that were not identified by lower resolution assays, however parental follow-up was not obtained and they were therefore excluded from the analysis. One of the 60 cases was found to be a false negative (DNA deletion corresponding to a single BAC clone at 16p13.1). The SNPs covering the $16 \mathrm{p}$ telomere region on 
the 260K NSP chip were not sufficiently dense to reach the criteria for abnormal. Another case was negative by the SNP array and positive by aCGH, but has not been confirmed. Eight samples tested for reproducibility were remarkably consistent among duplicate runs as well as $\mathrm{kb}$ size. The extremely high resolution platform makes secondary confirmation of real copy number changes superfluous. However, due to the sensitivity well within the normal range of variation, parental followup to differentiate familial CNVs from de novo changes are likely to be extremely important. This array not only provides exact base pair boundary determination for dosage changes, it also allows additional confirmation of deletion intervals through $\mathrm{LOH}$ and detection of UPD. In conclusion, the results from the whole genome SNP array were highly sensitive, specific and reproducible for the detection of significant DNA copy number changes.

\section{8 \\ Concept Design for a Uniform, Evidence-Based Molecular Karyotype and Shared Database}

D.H. Ledbetter ${ }^{a}$, A.R.Brothman ${ }^{b}$, C.L. Martin ${ }^{a}$

aDepartment of Human Genetics, Emory University School of Medicine, Atlanta, GA, 'b Departments of Pediatrics, Human Genetics, and Pathology, University of Utah School of Medicine, Salt Lake City, UT, USA

There is an explosion of new molecular technologies for genomewide, high resolution detection of gene dosage alterations, including aCGH using BAC clones or oligonucleotides, and quantitative SNP microarrays and beadchips. All of these technologies offer significant advantages over conventional karyotyping in the evaluation of children with unexplained mental retardation, and can be collectively referred to as Molecular Karyotype analysis. In addition to multiple technology platforms, there is a proliferation of different molecular karyotype designs representing 'home-brew' or custom-designed tests by individual CLIA laboratories. This diversity of designs and claims for clinical utility can be confusing to clinicians and poses significant challenges for assuring quality control in this rapidly evolving segment of genetic testing. Analogous to the successful effort to develop a Uniform Panel for Newborn Screening in the United States, we think it is possible to develop a process for an evidence-based, Uniform Molecular Karyotype design. We have engaged a group of experts in clinical genetics, clinical cytogenetics, clinical molecular genetics and bioinformatics to address the feasibility of developing a Uniform Molecular Karyotype as well as a Shared National Database for pathogenic and benign gene dosage imbalances. The major areas of discussion and consideration have been to: 1) Define what the intended use and clinical utility of a Molecular Karyotype should be and develop an evidence-based process for a uniform design. This design will be technology platform independent, with the common data output as genome sequence coordinates of copy number gains or losses. The initial clinical utility will be defined in terms of postnatal testing of children with unexplained developmental delay/mental retardation. Mechanisms will be developed for broad-based community input to nominate additions, deletions or changes in the Uniform Molecular Karyotype design/content with expert panel review. 2) Develop guidelines for a shared database for clinical laboratories performing Molecular Karyotyping to accelerate the empiric data acquisition and 'data mining' process to create a Human Gene Dosage Map by differentiating pathogenic vs. benign gene dosage imbalances. This database would be complementary to the Database of Genomic Variants (http://projects.tcag.ca/variation/) which represents data from normal populations. 3) Address new training and educational requirements both for laboratory geneticists to perform Molecular Karytoyping as well as for clinicians utilizing and interpreting such tests in their clinical practice. These needs include understanding the technology capabilities and limitations, but more importantly the required skills in genomics and bioinformatics necessary for appropriate clinical interpretation.

\section{9 \\ Integrating Genome-Wide Segmental Aneuploidy Profiling and Karyotyping in Cytogenetic Diagnosis of Patients with Idiopathic Mental Retardation and Congenital Abnormalities}

\section{Poot, R. Hochstenbach}

Department of Medical Genetics, University Medical Center

Utrecht, Utrecht, Netherlands

During the ten-year period of 1996-2005, a grand total of 36,325 patients with multiple congenital anomalies and mental retardation (MCAMR) were karyotyped, and subjected to FISH testing for specific microdeletion syndromes or subtelomeric imbalances in the eight laboratories for clinical cytogenetics in The Netherlands. Thus, 3,126 abnormal cases were identified ( $8.6 \%$ of the patients investigated). Using $1 \mathrm{Mb}$ resolution array platforms 11 studies of 623 selected patients with normal karyotypes reported in the literature identified segmental aneuploidies in 84 of them (13.5\%), while 4 studies using arrays with probe spacing of 30 to $70 \mathrm{~kb}$ identified $34(17.3 \%)$ abnormalities in selected patient populations and 25 abnormalities in 204 unselected patients (12.4\%). Use of targeted arrays with unselected patient populations allowed detection of abnormalities in $7.2 \%$ of cases. While these results clearly emphasize the merit of array-based techniques early during diagnostic workup of MCAMR it should be borne in mind that 423 of the chromosomal anomalies found in 36,325 patients (1.16\%) would not have been detected by BAC, oligonucleotide or SNP array platforms. These include apparently balanced, reciprocal translocations (171 cases), Robertsonian translocations (44 cases), apparently balanced inversions (114 cases), mosaicism with $<20 \%$ abnormal metaphases (including 43 cases of autosomal mosaicism and 39 cases of sex-chromosome mosaicism), and 69,XXX triploidy (12 cases). We conclude that concomitant karyotyping and genome wide array-based screening will afford a $20 \%$ detection rate of potentially pathogenic chromosomal aberrations, thus making MLPA, FISH and MAPHbased targeted techniques superfluous and cutting short a 'diagnostic odyssey'.

\section{0 \\ Discovery of New Syndromes by Array CGH and Understanding the Clinical Implications}

\section{L.G. Shaffer}

Signature Genomic Laboratories, Spokane, WA, USA

The wide-spread use of array CGH has altered the means by which new microdeletion syndromes are identified. Traditionally, the cytogenetic basis of microdeletion syndromes has depended upon the opportune ascertainment of a patient with a chromosomal rearrangement visible by G-banding who has an established clinical diagnosis. Array CGH has enabled the identification of novel, recurrent imbalances in patients whose clinical features did not fit a known genetic syndrome. Using this 'genotype-first' approach, we have reported the identification of the novel 16p11.2p12 and 1q41q42 microdeletion syndromes. In addition, with over 17,000 patients now tested in our laboratory with array CGH and deposited into our database, we have identified many other recurrent, novel abnormalities in two or more patients. However, unlike the 'phenotype-first' approach of traditional cytogenetics, describing a new 'syndrome' based on the molecular alteration has posed some problems. With the phenotype-first approach, a constellation of features constitutes a new syndrome. Patients with this 'classic' presentation will be labeled with the syndrome and the search for the molecular or cytogenetic etiology can ensue. In the genotype-first approach of array CGH, the patients' clinical features may vary widely; patients may not share typical features and may exhibit the complete spectrum of the disorder. This makes describing a syndrome more difficult and complicates the identification of additional 
patients. One approach is to identify patients with de novo aberrations. However, the inheritance of a chromosomal gain or loss does not always predict that the alteration is a benign variant. The classic example is the deletion of 22q11.2 which often can be inherited from a mildly affected parent. We have many examples of the same aberration being inherited from an apparently unaffected parent in some families and de novo in others. This complex inheritance further confounds the assignment of the aberration as clinically relevant or a benign CNV. The identification of additional patients and the delineation of the genes that contribute to the phenotypes will aid in the categorization of such alterations identified by microarray analysis.

\section{1 \\ Laboratory Quality Improvement and Financial Success through Six Sigma Methodology \\ S.M. Zneimer \\ Department of Biology, Moorpark College, Moorpark, CA, and \\ MOSYS Consulting, Los Angeles, CA, USA}

Six sigma methodology provides the means to recreate laboratory processes so that errors and defects in a system never arise. Six sigma gives laboratories the tools to improve costs through continuous quality improvement. Six sigma is defined as 3.4 errors in one million opportunities. Yet, most laboratories and major companies operate at approximately 3 sigma, which equals 67,000 errors in a million opportunities. By implementing six sigma methods into the laboratory, one can see financial benefits through continuous quality improvement that will ultimately lower overall costs with the elimination of waste and errors in the system. Three examples of six sigma research projects were conducted which succeeded in producing positive effects in overall quality, productivity and costs. The first study analyzed cytogenetic and FISH tests to establish a baseline formula for easy, reproducible determination of cost of testing in the laboratory. The second study determined baseline microscope analysis productivity and improved efficiency and quality with process improvements and the elimination of wasted steps and errors. Results showed improvement of microscope productivity to a minimum of 5 cases/day/tech for constitutional cytogenetic microscope analysis. The third study analyzed Her2 FISH testing to decrease cost per test and improve rate of success of hybridizations. Results showed a 30\% decrease in rework time and $43 \%$ improvement in cost of testing. In each of these three studies, laboratory improvements were made that can be reproduced in other laboratories through six sigma methodology.

\section{2 \\ Is Unreported Chromosomal Variation in Hyraxes Indicative of Cryptic Speciation?}

\section{M.L. Houck, S.J. Charter, L.G. Chemnick, H.A. Davis, J.A. Fronczek, A. Johnson, O. A. Ryder \\ Zoological Society of San Diego, Conservation and Research for Endangered Species, Escondido, CA, USA}

Hyraxes are rabbit-sized herbivorous mammals occurring in rocky areas, open grassland, and forested areas in southwestern Asia and most of Africa. They belong to the order Hyracoidea which consists of a single family, Procaviidae, comprised of three extant genera and 7-14 species. Two of the genera are primarily terrestrial and diurnal (Procavia and Heterohyrax) while the third is mainly arboreal and nocturnal (Dendrohyrax), but overlapping habitats and slight morphological differences can hinder identification. Although there has been controversy regarding the closest living relatives of Hyracoidea, analysis of DNA sequences places them in the Superorder Afrotheria along with five other orders whose representatives include elephants, manatees, aardvarks, elephant shrews, and tenrecs. Previously published karyotypes of Hyracoidea report the diploid number as $2 n=54$ for all three genera. The G-band patterns are largely comparable for Procavia capensis and Heterohyrax brucei, but additional heterochromatic arms distinguish the single Dendrohyrax arboreus karyotype. We report $2 \mathrm{n}=52$ and $2 \mathrm{n}=56$ hyrax karyotypes for the first time, from individuals submitted as $P$. capensis. A fission/fusion event is involved in the $2 \mathrm{n}=52$ vs $2 \mathrm{n}=54$ individuals while microchromosomes contribute to the $2 n=56$ karyotypes. Mitochondrial cytochrome b sequences from the $2 \mathrm{n}=52$ animals did not closely match either of the sequences available in GenBank (P. capensis, $93 \%$ identity and H. brucei, $91 \%$ identity). Sequencing of the $2 \mathrm{n}=56$ animals is in progress. Our findings may support the postulated genetic distinction between the northwestern and south central populations of $P$. capensis based on mitochondrial DNA RFLPs or could indicate previously unreported taxa. A lack of pedigree and capture location information for purported $P$. capensis makes the observed karyotypic variation difficult to explain. Additional molecular and cytogenetic studies on animals from known provenance are necessary to evaluate if genetic differences reflect cryptic taxa or variation within a single taxon.

\section{3 \\ Chromosomal Aberrations Associated with Congenital Abnormalities, Infertility, and Repeated Early Embryonic Loss in the Domestic Horse (Equus caballus)}

\section{T.L. Lear, J. Lundquist}

Molecular Cytogenetics Laboratory, Maxwell H. Gluck Equine Research Center, Veterinary Science Department, University of Kentucky, Lexington, KY, USA

Cytogenetic analysis is an important diagnostic procedure for identifying chromosomal aberrations that cause congenital abnormalities, infertility and repeated early embryonic loss in the domestic horse (Equus caballus; ECA). Most equine samples are submitted to rule out chromosomal abnormalities that cause infertility. Our equine clinical cytogenetics service has identified numerous cases involving sex chromosome abnormalities, including XY Sex-Reversal Syndrome, XX SexReversal Syndrome, and Equine Turner Syndrome [63,X and 64,X, del(Xp)]. A previously unreported case of autosomal trisomy $(65, \mathrm{XY}$, +31 ) was identified in a newborn foal with severe congenital abnormalities. More recently, four new autosomal translocations were identified in mares with repeated early embryonic loss $[64, \mathrm{XX}, \mathrm{t}(1 ; 16)$; $64, \mathrm{XX}, \mathrm{t}(1 ; 21) ; 64, \mathrm{XX}, \mathrm{t}(16 ; 22) ;$ and $64, \mathrm{XX}, \mathrm{t}(4 ; 13)]$. In three of these cases, the newly assembled horse genome sequence was used to identify gene markers surrounding the breakpoints. BACs containing these gene markers were then FISH mapped to characterize the translocations. In other horses chromosomal polymorphisms were identified, such as variation in centromeric heterochromatin and in the heterochromatic band at ECAXq21. Overall, chromosomal aberrations were identified in approximately $25 \%$ of the samples submitted for karyotyping. In the future, equine SNP chips may enable the identification of some chromosomal abnormalities beyond the resolution of conventional cytogenetic methods.

\section{4 \\ Experience with a Microarray Designed Specifically to Characterize Marker Chromosomes}

C.D. Kashork, B.C. Ballif, L.G. Shaffer

Signature Genomic Laboratories, Spokane, WA, USA

By comparing DNA from a patient directly with that of a known control, array comparative genomic hybridization (CGH) can detect gains or losses of DNA segments associated with chromosomal abnormalities. A loss of material typically indicates a deletion which is relatively straightforward to interpret. However, a gain of a DNA segment may have a more complex interpretation. Increased copy number of a chromosome segment may indicate a duplication; however, copy-num- 
ber gains may result from other chromosome rearrangements, including marker chromosomes. We present our experience with 58 marker chromosomes identified in our laboratory. After identification of a gain by chromosome analysis or the SignatureChip ${ }^{\circledR}$, we used our MarkerChip ${ }^{\mathrm{TM}}$, designed specifically to target the most proximal unique chromatin in the pericentromeric regions, to further characterize the markers. In a few cases, we used the SignatureChip ${ }^{\circledR}$ WG, which has incorporated clones from the MarkerChip among the $>4,600$ clones on the array. FISH with BAC clones used in the construction of the microarrays was an important part of the complete assessment of the marker chromosomes. The most common nonacrocentric markers identified involved the short arm of chromosome $12(\mathrm{n}=4)$; the short arm of chromosome $17(\mathrm{n}=4)$; and chromosome 8 , involving only the short arm $(n=3)$, only the long arm $(n=2)$, or both arms $(n=4)$. The most common acrocentric markers involved $15 q(n=9)$ and $22 q(n=$ 8). Some low-level mosaics could not be identified. In addition, because we avoided the highly repetitive heterochromatic regions of the pericentromeres, markers composed of little or no euchromatic DNA could not be identified. Nonetheless, the establishment of microarrays arms the cytogeneticist with the tools to identify and delineate marker chromosomes in an efficient and comprehensive manner.

\section{5}

\section{Novel Genomic Syndromes Defined by Array CGH Are Focused in Regions of High Complexity}

S. Aradhya, A.G. Mitchell, S. Warren, J. Dorson, G. Richard

GeneDx, Gaithersburg, MD, USA

In a cohort of 950 cases referred for whole-genome oligonucleotide array CGH, we have identified $141(\sim 15 \%)$ positive cases and $95(\sim 10 \%)$ cases of variants of unknown clinical significance. Many of the positive cases involve rearrangements in regions of high genomic complexity featuring a rich concentration of segmental duplications. We have found such rearrangements in 1q21, 2p15, 8p23.1, 9q34, 16p11, 16p13, and $17 \mathrm{q} 21$, and all of these loci were recently linked to disease-causing syndromes. As expected, both deletions and reciprocal duplications have been identified at many of these loci, but not all rearrangements were pathogenic. We have also identified other rearrangements that have not been reported but are likely causative of clinical phenotypes. These were found in 10q23-q24 and 16q24.2-q24.3. The variants of unknown clinical significance, in contrast, are generally not found in regions of high complexity, and sometimes involve only a few genes. However, a number of them have proved to be de novo occurrences and may have clinical significance. Our findings endorse the logic of focusing array probes in all regions of high complexity to identify diseasecausing syndromes, and also to uncover novel ones. However, the de novo cases of variants of unknown significance emphasize that the rest of the genome also requires a reasonable degree of coverage, at least until collective data from array CGH studies point the way to a comprehensive array design to specifically detect all disease-causing unbalanced genomic rearrangements.

\section{6}

Expansion in Size of a Terminal Deletion: A Paradigm Shift for Parental Follow-up Studies

S.T. South ${ }^{a, d}$, A.F. Rope ${ }^{a}$, A.N. Lamb ${ }^{a, c, d}$, E. Aston ${ }^{a}$, N. Glaus ${ }^{a}$, H. Whitby ${ }^{a}$, T. Maxwell ${ }^{\text {, X.L. Zhu }}$, A.R. Brothman ${ }^{a-d}$

Division of Medical Genetics, Departments of a Pediatrics, ${ }^{b}$ Human Genetics, and 'Pathology, University of Utah, ${ }^{\mathrm{d} A R U P}$ Laboratories, Salt Lake City, UT, USA

Background: Parental studies are often necessary subsequent to the identification of a chromosome abnormality. The recommended studies are based on assumptions about how chromosome rearrangements occur. One such assumption is that deletion size is stable through generations.

Results: We have identified a family where a small subtelomeric deletion in a phenotypically and cytogenetically normal mother expanded nearly 10 -fold into a clinically consequential and cytogenetically visible deletion in her affected daughter.

Conclusion: Traditional parental follow-up studies would have not identified this expansion, but would have rather classified the deletion in the daughter as either de novo or benign. Only by sizing the deletion by array CGH in both the mother and the daughter was the expansion recognized. Previous assumptions about chromosome behavior suggest that this phenomenon may have been easily missed in other cases of chromosomal deletions. Therefore, this case illustrates the need for more comprehensive analyses of parental chromosome structure when characterizing an abnormality in a child.

\section{7 \\ Dissecting Microdeletions to Find the Haploinsufficient Genes \\ U. Francke \\ Departments of Genetics and Pediatrics, Stanford University School of Medicine, Stanford, CA, USA}

Recurrent microdeletions, and corresponding microduplications, arise by a mechanism of non-homologous recombination between short segmental duplications that flank the common deletion regions. To dissect the complex associated phenotypes and to determine which genes are dosage-sensitive and potentially haploinsufficient, my laboratory has used two approaches: (1) identify and study rare patients with unusual deletions or translocations of the region; and (2) use chromosome engineering technique to create partial deletions in mouse models. The examples to be presented include (a) the Williams-Beuren syndrome deletion at 7q11.23, and two adjacent partial deletions of the conserved region on mouse chromosome band 5G2; and (b) the PraderWilli syndrome deletion at $15 \mathrm{q} 12$, and a $150 \mathrm{~kb}$ deletion of mouse chromosome band $7 \mathrm{C}$ that contains all $>40$ copies of the $\mathrm{C} / \mathrm{D}$ box small nucleolar RNA cluster Snord116 (Pwcrl/MBII-85). This snoRNA cluster was implicated by studies of PWS patients with translocations or smaller deletions. General conclusions from our studies of the mouse models are: (1) deletion mice have phenotypes that recapitulate parts of the human syndromes; (2) expression levels of deleted genes do not always follow copy number; (3) non-deleted genes close to the breakpoints may have altered expression due to long-range effect of the chromatin rearrangement.

\section{8 \\ From Genome to Gene: Integration of High Resolution Whole Genome SNP Oligonucleotide Microarray Analysis (SOMA) into the Clinical Cytogenetics Laboratory}

\section{B. Levy, V. Jobanputra, O. Nahum, D. De Vivo, D. Warburton}

Columbia University Medical Center, New York, NY, USA

Currently our ability to predict the clinical consequences of a constitutional chromosome imbalance is limited by the imprecise manner in which most abnormalities have been defined in the past. Prognostic information derived empirically by reviewing 'similar' cases in the literature is inexact because it will combine cases that have very different true genetic imbalances. High resolution SNP oligonucleotide microarray analysis (SOMA) allows for much more precise definition of the boundaries and nature of the region of imbalance, including the gene content. To validate the use of the Affymetrix 500k array for this 
purpose, we carried out a blinded study of specimens with known cytogenetic aberrations, including cryptic subtelomeric unbalanced rearrangements, as well as patients with diseases due to single gene abnormalities. In all cases, we reliably detected the chromosomal imbalances, with sizes ranging from single genes to several megabases. We have also used SOMA in a clinical setting to characterize more precisely the size and gene content of visible cytogenetic aberrations or those previously defined only by FISH. In a few instances, the cytogenetic aberration was far more complex than indicated by GTG banding. By this approach, we also uncovered a new microdeletion syndrome involving patients with GLUT-1 deficiency. Other candidates for SOMA included patients with non-specific phenotypic abnormalities and apparently balanced karyotypes. In this manner, we unexpectedly discovered a $3.8 \mathrm{Mb}$ deletion in the long arm of chromosome 20 in a patient with a non-standard balanced $t(11 ; 22)$ translocation. The goal of the new cytogenetics should be the development of a genotype-phenotype map describing the effects of gene imbalance for each precisely defined chromosome segment. Ultimately this should lead to a new understanding of the role of individual genes in development, as well as of gene interactions when multiple genes are involved.

\section{9 \\ Deletion, Duplication and Copy Number Neutral Loss of Heterozygosity of Chromosome 22 Revealed by High Density SNP Array Analysis of Pediatric Rhabdoid Tumors}

J. Biegel ${ }^{a}$, A. Sievert ${ }^{a}$, X. Gai ${ }^{c}$, H. Hakonarson ${ }^{a}$, T. Shaikh ${ }^{a}$, E. Jackson ${ }^{b}$

aDepartments of Pediatrics and 'Neurosurgery, The Children's Hospital of Philadelphia and the University of Pennsylvania School of Medicine, and 'Center for Biomedical Informatics,

The Children's Hospital of Philadelphia, Philadelphia, PA, USA

Malignant rhabdoid tumors (MRT) are rare, highly aggressive neoplasms found most commonly in infants and young children. Although they may present in any location in the body, they are predominantly found in the central nervous system and kidney, comprising approximately $1-2 \%$ of infant brain and renal tumors. The INI1/hSNF5/ SMARCB1/BAF47 gene in 22q11.23 has been implicated in the development of MRT. Previous studies have suggested that approximately $75 \%$ of rhabdoid tumors have a detectable mutation/deletion of INI1. To date, no other mechanism has been elucidated to explain the remaining $25 \%$ of cases. In the present study, the Illumina 550K BeadChip SNP array was used to detect copy number changes and loss of heterozygosity in genomic DNA from 50 rhabdoid tumors ( 37 brain, 2 spinal cord, 5 renal, 6 extra-renal). Results were analyzed using Beadstudio software provided by Illumina and in-house analysis tools developed to identify copy number variation. Forty-seven of 50 tumors had detectable aberrations involving 22q11.23, including 15 tumors with heterozygous deletions, 18 tumors with homozygous deletions, and 14 tumors with copy number neutral loss of heterozygosity. Several cases had complex alterations of chromosome 22 in addition to the deletions in the 22q11.2 region. The majority of tumors had a variable number of potential disease associated copy number variations but none were consistently found across multiple patients. The remaining 3 of 50 cases did not have detectable abnormalities of $22 \mathrm{q} 11.2$ by the array analysis, however interphase FISH and/or MLPA analysis revealed alterations of INII that may have been below the resolution of the array. The present results suggest that INI1 is the primary gene implicated in the development of pediatric rhabdoid tumors. A combination of approaches utilizing high density arrays, FISH, MLPA and INI1 sequence analysis can be used to identify the majority of alterations of INI1 that lead to the development of rhabdoid tumors.

\section{0 \\ Identification of Commonly Aberrant Genomic Regions Using High Resolution Oligo Array CGH of FFPE Breast Cancer Samples}

C.E. Carmack ${ }^{a}$, R. Davis ${ }^{b}$, A. De Witte ${ }^{a}$, B. Poirier ${ }^{b}$, E. Lin $^{a}$,

A. Borowski ${ }^{b}$, J. Ghosh ${ }^{a}$, J. Gao ${ }^{a}$, S. Giles ${ }^{a}$, E. LeProust ${ }^{a}$, D. Amorese ${ }^{a}$, D. Roberts ${ }^{a}$, S. Shams ', J. Gregg ${ }^{b}$

${ }^{a}$ Agilent Technologies, Santa Clara, bUniversity of California, Davis, 'BioDiscovery Inc., El Segundo, CA, USA

Several common chromosomal aberrations have been identified in breast cancer. For instance, amplification of the portion of $17 \mathrm{q}$ encompassing the ERBB2 oncogene has been estimated to be present in 10$34 \%$ of breast cancer cases. Other aberrations, especially common in estrogen receptor (ER) positive cases include loss of heterozygosity at $16 \mathrm{q}$ and gain at 1q. It is important to accurately delineate copy number transitions and boundaries to define the genes that consistently lie within aberrations. Comparative genomic hybridization provides a means to determine the changes in genomic copy number and transition points. We applied high resolution microarray-based (aCGH) analysis of formalin fixed paraffin embedded invasive ductal breast carcinoma samples. We used a $244 \mathrm{~K}$ CGH array which consists of 244,000 in situ synthesized 60 -mer oligonucleotide probes on a single $1^{\prime} \times 3^{\prime}$ slide. This feature density results in an average genomic distance between probes of $\sim 6 \mathrm{~kb}$ across the entire human genome. We analyzed 58 invasive ductal carcinomas, which included 18 erbB2 positive (3+ immuno and/or FISH positive), 7 erbB2 2+ positive (via immunohistochemistry), 24 ER+ and 34 ER-, 6 Grade I, 25 Grade II, and 27 Grade III tumors derived from archival formalin fixed paraffin embedded (FFPE) tissue from UC Davis. Altered genomic regions were identified using Nexus 2.0 with BioDiscovery's Rank Segmentation algorithm. Class comparisons, using FDR correct Fisher Exact Testing was utilized on the samples. Significant FDR corrected regions were identified for classification of grade of tumor, ER status, and outcome. Unsupervised hierarchical clustering identified 3 different classes of samples based on their CGH profile, two of which belonged to a poor prognosis/survival outcome class and one that belonged to a good prognosis/survival outcome class. Lastly, the CGH profiles were able to accurately identify the erbB2 amplification structure, including Top2A co-amplification, as it is correlated with better disease free survival with anthracycline based therapy.

\section{1 \\ Homozygous Deletion of Copy Number Variants Detected by CGH Microarray as a Potential Mechanism for Mental} Retardation

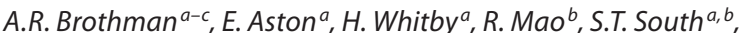 \\ C. Curry ${ }^{d}$
}

aDepartment of Pediatrics, ${ }^{\text {bP }}$ Pathology and ARUP Laboratories, 'Human Genetics, University of Utah School of Medicine, Salt Lake City, UT, USA, ${ }^{\mathrm{d}}$ Genetic Medicine Central California/University of California San Francisco, CA, USA

We describe two cases of homozygous deletions detected by array CGH analysis in patients referred for study due to mental retardation and other dysmorphic features. Analysis performed by routine methods using the Spectral Genomics/Perkin Elmer SpectralChip $2600^{\mathrm{TM}}$ BAC microarray platform revealed broad separation seen by dye swap of a single BAC clone in each patient. Both cases were confirmed by FISH using the same BAC observed to be abnormal in the microarray. Both patients were offspring of non-consanguinous parents, and both patients had multiple previous studies (all with normal results), including cytogenetics and subtelomeric FISH. Patient 1 had microcephaly, distinctive craniofacial features, brachymetacarpia, brachymetatarsia, and severe mental retardation; he was born to phenotypically normal 
parents. Array CGH analysis showed an interstitial deletion at a commonly observed benign copy number variant (RP11-17M8 at 8q24.2); this was confirmed by FISH to be deleted on both chromosome 8 homologs. Heterozygosity for the deletion was found in the mother and uniparental disomy was excluded. The father was unavailable for analysis. An Agilent 244K oligonucleotide array demonstrated a homozygous deletion of approximately $1.2 \mathrm{Mb}$, spanning a gene poor region at 8q24.2. Patient 2 had a homozygous interstitial deletion including a BAC that was not reported in public or internal databases (RP11-89P15 at 12q21.1). He has mild craniofacial dysmorphic features, bifid uvula, peripheral pulmonic stenosis and developmental delay. An Agilent $244 \mathrm{~K}$ array indicated that this was a $0.79 \mathrm{Mb}$ deletion; one highly conserved gene maps within this region, but has no known function. Since heterozygosity for this deletion was confirmed in both parents and two sisters (all with normal phenotypes), we are defining this as a benign copy number variant in heterozygous form. A younger brother with mild developmental delay is also homozygous for this deletion. The findings in these two patients may represent a new mechanism for mental retardation which will mimic an autosomal recessive disorder We suggest that analysis of copy number variants in phenotypically abnormal patients should thus include scrutiny for homozygosity.

\section{2}

\section{SNP Arrays Open a Window on Complex Mosaicism}

\section{L.K. Conlin, B.D. Thiel, L. Medne, L. Campbell, J.T. Glessner,} C. Bonnemann, I.D. Krantz, H. Hakonarson, N.B. Spinner

Department of Genetics, Children's Hospital of Philadelphia, Philadelphia, PA

The use of array technology in the cytogenetics laboratory has enormously enhanced our ability to detect small genomic deletions and duplications, and additionally, to identify low-level mosaicism. The Illumina SNP array system is particularly informative for these studies because of the combination of analysis of signal intensity and allele frequencies. In addition, the allele frequencies can be used to infer inheritance and can be used to find evidence for uniparental disomy. We have derived an algorithm to calculate the percent mosaicism based on the altered B allele frequencies observed.

We have used the Illumina HumanHap550 SNP array on a series of 180 patients who presented with various clinical abnormalities with either known or suspected chromosomal abnormalities. We identified four patients with evidence for multiple cell populations. In two of these patients, initial chromosome analysis did not reveal the mosaicism but we were able to subsequently confirm it by molecular cytogenetic analysis after identification using the SNP array. One patient had mosaic trisomy 14 with 2 distinct patterns of allele frequencies and subsequent cytogenetic analysis revealed trisomy 14 in $5 \%$ of cells. A second patient had mosaic duplication of the short arm of chromosome 12 , in addition to a previously identified deletion of 22q11. A third patient had three cell lines, which included one with a supernumerary inverted dup(15), another with a supernumerary del(15), and a cytogenetically normal cell line. A fourth patient was a phenotyically normal male who was mosaic XX/XY. This last patient was the most unusual, as the array data suggested that the patient was a chimera with uniparental disomy in the XX cell line, which we believe is consistent with imprinting abnormalities that can be associated with his clinical abnormalities.

SNP array technology is revolutionizing our ability to detect mosaicism, indicating a new level of complexity in the types of chromosome abnormalities that contribute to human disease.

\section{3}

\section{Fetus in Fetu: An Unusual Case}

A.M. Cherry a , I. Schrijver ${ }^{a}$, J. Kaplan ${ }^{b}$, A. Kwan ${ }^{b}$, D. Perry ${ }^{a}$, J.T.C. Shieh ${ }^{b}$

Departments of aPathology and bediatrics, Division of Clinical Genetics, Stanford University School of Medicine, Stanford, CA, USA

Fetus in fetu is a rare anomaly with fetiform masses found inside a developing fetus, living child or adult. It has been presumed that these masses represent an error in monozygotic twinning. It is a rare occurrence, having only been described in the literature in less than 40 cases. Typically, there is only one twin, however there have been some reported cases with more than one.

Here, a 30-week gestation female fetus was found on prenatal ultrasound to have intra-abdominal fetal elements. Following delivery, the child demonstrated a grossly distended abdomen and 11 fetiform masses were found within the peritoneum and retroperitoneum. Of these masses, four had vertebral columns, eight had recognizable limbs and/or appendages and three had neither but did have complex tissue architecture. In addition to these fetiform masses, a mature ovarian teratoma was found. Five of these masses were karyotyped, as was the infant. All had a normal female 46,XX karyotype. Genotype testing should determine the relationship of these masses to the infant.

\section{4 \\ Cytogenetic Variation in Populations of Cope's Gray Tree Frog Hyla chrysoscelis}

\section{J.E. Wiley, D.S. Bonner}

Department of Pediatrics, Division of Medical Genetics, Brody School of Medicine, East Carolina University, Greenville, NC, USA

Cope's gray tree frog, Hyla chrysoscelis, is endemic to North America and has a range that is bordered on the east by the Atlantic Ocean, extends north into Canada, south to the Gulf of Mexico as well as the northern half of Florida, and west to approximately the middle of the continent. In a population this large genetic heterogeneity is expected and has been documented for this species by a number of techniques, such as restriction enzyme polymorphisms, immunological distance, mitochondrial DNA sequencing, and cytogenetics. Some of these studies have used specimens of $H$. chrysoscelis that were collected from one or two localities in the range of the species while others sampled the population more broadly. $\mathrm{H}$. chrysoscelis is polymorphic for the chromosome location of the $18 \mathrm{~S}$ and $28 \mathrm{~S}$ ribosomal genes and we have identified five cytogenetically distinct populations of the species using silver-staining of the nucleolar organizing regions. We conducted targeted collections of animals from the distinct populations and performed cytogenetic analysis using silver-staining (to document population of origin), C-banding, and replication banding. These studies revealed cytogenetic variation, in accordance with the data originally derived from silver-staining, which supports the existence of five cytogenetically distinct populations of $H$. chrysoscelis. 


\section{5}

The Influence of Genetics, Epigenetics, and Environment on Acquired Chromosomal Abnormalities: A Twin Study

K.S. Haydu, P. Vitazka, J.-U. Kang, A. Ferreira-Gonzalez,

C.K. Jackson-Cook

Department of Pathology, Virginia Commonwealth University, Richmond, VA, USA

Although acquired chromosomal changes have been implicated in several age-related conditions, little is known about their incidence or etiology. For example, are individuals genetically predetermined to acquire specific chromosomal changes or do these chromosomal changes arise as a consequence of environmental exposures? To answer this primary question we determined the chromosome-specific frequency of acquired chromosomal changes in identical (MZ) and fraternal (DZ) twins. To date, 51 healthy individuals, ranging in age from 9 to 80 years, have been studied including 20 complete twin pairs and 11 single twins. Acquired chromosomal changes were assessed from lymphocytes by scoring the frequency of micronuclei $(\mathrm{MN})$ observed in 1000 cytokinesis-blocked binucleate cells/subject. Chromosome-specific frequencies of MN were determined using our novel spectral karyotyping (SKY) assay. The overall frequency of MN observed ranged from $0.1 \%$ to $4.6 \%$. The mean frequency of MN was not significantly different in the 29 males studied $(1.44 \% \pm 0.18)$ compared to the 22 females evaluated $(1.58 \% \pm 0.22)$. As expected, a significant increase in $\mathrm{MN}$ frequencies was observed with increasing age $(\mathrm{p}<0.005)$. The $\mathrm{DZ}$ twins (who share $1 / 2$ their genes) showed a 2.3 -fold increased difference in their MN frequencies compared to the identical MZ twin pairs. However, the MZ co-twins also demonstrated differences in MN frequencies, suggesting that both genetics and environmental exposures are contributory factors to the observed variation in $\mathrm{MN}$ incidence. Indeed, a history of smoking was shown to significantly increase overall micronuclei frequencies $(\mathrm{p}<0.005)$. The chromosome-specific SKY assay showed that the majority $(92.3 \%)$ of $\mathrm{MN}$ contained chromatin from a single chromosome, with $7.7 \%$ of the $\mathrm{MN}$ containing chromatin from 2 or more chromosomes. A non-random pattern of chromosomal exclusion into micronuclei $(\mathrm{p}<0.005)$ was detected, with $26 \%$ of all MN containing X chromatin, $13 \%$ Y chromatin, and 53\% autosomal chromatin. Within the autosomal subset of $\mathrm{MN}$, a non-random pattern of exclusion $(p<0.005)$ was also observed, with chromosomes $1,3,4$, 8 , and 9 having significantly higher frequencies and chromosomes 17 , $19,20,21$, and 22 significantly lower. To determine if the presence of differentially methylated chromatin influences chromosomal instability, additional studies were done using amplification of inter-methylated sites (AIMS) after digestion with the isochizomer pair SmaI-XmaI, followed by comparative genomic hybridization (CGH). The global methylome profile identified from these studies showed a generalized R-band like pattern, but presented with brighter staining on regions of distal $1 \mathrm{p}$, the acrocentric short arms, and chromosomes 19 and 22. Also, targeted areas of intense staining were observed at bands Xq22; $1 \mathrm{p} 36.3 ; 1 \mathrm{q} 42 ; 7 \mathrm{p} 22 ; 8 \mathrm{q} 13 ; 8 \mathrm{q} 22 ; 14 \mathrm{q} 24 ; 15 \mathrm{q} 11.2$; and 16p11.2. These methylome studies, using CGH following co-hybridization of the AIMS products from younger and older identical co-twins, will further clarify the degree of variation in these patterns that is acquired with age and their correlation, if any, to the observed patterns of acquired chromosomal instability. Collectively, the information gained from this study provides insight as to the magnitude of variation in the frequency of acquired chromosomal changes between individuals (up to 46-fold differences observed) and has allowed for the recognition that both genetic and environmental factors contribute to this variation. This knowledge is important for developing/optimizing health screening assays for age-related conditions that might arise from the exploitation of knowledge regarding acquired chromosomal aberrations.

\section{6 Concurrent Homozygous $\alpha$-Thalassemia and Trisomy 7
Mosaicism: Case Report of a Liveborn Male Infant}

F. Quintero-Rivera ${ }^{a, d \#}$, P. Abreu-e-Lima ${ }^{b \#}$, I.H. Zhang ${ }^{c}$, F. Bieber ${ }^{a, b}$, M.M. Parast ${ }^{b}$

aDepartment of Pathology, Division of Cytogenetics, Brigham and Women's Hospital, 'Department of Pathology, Division of Women's and Perinatal Pathology, 'Department of Pediatrics, Division of Pediatric Hematology/Oncology, Dana Farber Cancer Institute, Boston, MA, USA, dDepartment of Pathology \& Laboratory Medicine, David Geffen School of Medicine at UCLA, LA, CA, USA

We report a male infant born at 35 weeks gestational age with an atypical presentation of homozygous $\alpha$-thalassemia. The liveborn infant displayed abnormalities of the upper limbs and genitalia, which are vascular-type disruptive defects associated with this disease. Cardiomegaly and placentomegaly were the only evidence of fetal hydrops. Post-natal karyotype revealed mosaicism for trisomy 7, yet another rare finding in a liveborn. Finally, we discuss our institutional experience with each of these rare conditions and the potential contribution of each to the overall unusual clinical presentation in this patient. To our knowledge, this is the first such case report of simultaneous diagnoses of homozygous $\alpha$-thalassemia and trisomy 7 mosaicism.

\# These authors have contributed equally to this work.

\section{7 \\ Segregation of a Cryptic Familial Balanced Translocation (1p;22q) Resulting in Detection of Both Adjacent-1 Segregants}

D.O. Saul, S.N. Dowey, E.D. Wood, D.A. Batista, K.J. Blakemore, G. Stetten

Department of Gynecology and Obstetrics, Johns Hopkins University School of Medicine, Baltimore, MD, USA

A subtle translocation involving the terminal regions of chromosomes $1 \mathrm{p}$ and $22 \mathrm{q}$ was identified in a family by high resolution karyotyping, subtelomere FISH and array CGH. Segregation of the balanced $t(1$; 22)(p36.3;q13.3) chromosome translocation in this family resulted in two types of unbalanced chromosome complements. The proband inherited the derivative chromosome 1, resulting in partial monosomy for terminal $1 \mathrm{p}$ and partial trisomy for terminal 22q. He has features of both monosomy 1 p36.3 and trisomy 22q13 syndromes. Because of the familial translocation the couple chose to pursue in vitro fertilization with preimplantation diagnosis. Biopsied cells analyzed by FISH (at an outside laboratory) were predominately unbalanced for the translocation and/or aneuploid, and a pregnancy was not achieved. Subsequent natural pregnancies resulted in two fetuses that inherited the derivative chromosome 22 and another fetus that carried the balanced translocation. The pregnancies carrying unbalanced translocations were electively terminated, and the fetus with the balanced translocation was diagnosed with severe IUGR and anomalies at 20 weeks gestation. The etiology of the IUGR and fetal anomalies was thoroughly investigated and remains unknown. The balanced translocation has been studied with a high density BAC array (4200 clones) that failed to detect any imbalance. The extended family has a strong history of mental retardation and infant mortality. Therefore, it is not surprising that both adjacent-1 segregants were seen in viable pregnancies. This case underscores the importance of genetic counseling and appropriate testing for families that harbor rare but clinically devastating chromosomal rearrangements. The value of array CGH in prenatal diagnosis is discussed. 


\section{8}

Short Stature and Minor Anomalies in a Girl Having a

Maternal Inherited Derivative Chromosome 20 with an Interchromosomal Insertion of the 9p13.2-9p21.3 Segment Resulting in 16.6-Mb Interstitial 9p Duplication: Further Delineation of the Critical Region for the 9p-Duplication Syndrome

Y.S. Zou ${ }^{a}$, X. Huang ${ }^{a}$, M. Ito ${ }^{b}$, S. Newton ${ }^{c}$, J. Milunsky ${ }^{a-c}$

${ }^{a}$ Cytogenetics Laboratory, ${ }^{b}$ Molecular Genetics Laboratory,

'Medical Genetics, Center for Human Genetics, Boston, MA, USA

Although approximately 150 patients with partial or complete $9 p$ trisomy have been reported, limited studies have been conducted at the molecular level to characterize the critical region for the $9 p$-duplication syndrome. We studied a 12-year-old girl with short stature, language/speech delay, and physical findings including mild microcephaly, down-slanting palpebral fissures, prominent nasal root with a bulbous nasal tip, clinodactyly of the fifth finger, and delayed bone age, all of which are among the most common clinical manifestations of the 9 p-duplication syndrome. Her conventional chromosome study revealed a derivative chromosome 20 [der(20)ins(20;9)(p13;p13.2-p21.3)] with the 9p13.2-9p21.3 segment inserted into the short arm of chromosome 20 at band $20 \mathrm{p} 13$, resulting in an interstitial duplication of the $9 \mathrm{p}$ segment. FISH results of subtelomere and whole chromosome painting further confirmed these findings. 500K SNP-array genotyping revealed a duplication of $16.6 \mathrm{Mb}$ from 21.1 $\mathrm{Mb}$ to $37.7 \mathrm{Mb}$ of DNA from 9 pter and no copy number changes of chromosome 20. The mother carried a balanced insertion between chromosome 9 and 20, ins $(20$ 9)(p13;p13.2-p21.3). BAC FISH confirmed the breakpoints and the duplication of chromosome 9, supporting that some of the common phenotype of $9 \mathrm{p}$-duplication syndrome as we observed in this patient was caused by a duplication of $16.6 \mathrm{Mb}$ of the 9 p13.2-9p21.3 segment. However, for mental retardation and other $9 \mathrm{p}$-duplication facial findings, the breakpoints' location in this patient and previously reported cases suggest that the critical region lies within $4 \mathrm{Mb}$ of the $9 \mathrm{p} 22$ segment from $12 \mathrm{Mb}$ to $16 \mathrm{Mb}$ of DNA from 9pter.

Low copy repeats present at the breakpoints of chromosome 9 , might have originated the duplication of chromosome 9 segment by illegal recombinations.

\section{9}

Familial Translocation $(10 ; 12)$ with Deletion of 12q21.32

P. Jacky ${ }^{a}$, X. Li ${ }^{b}$, J. Sherman ${ }^{a}$, M. DeWitt ${ }^{a}$, O. Lamb ${ }^{a}$, C. Osborn ${ }^{a}$, V. Payne ${ }^{a}$, J. Trinklein ${ }^{a}$, D. Rappaport ${ }^{a}$, L. Linck ${ }^{a}$, D. Kostiner ${ }^{a}$

${ }^{a}$ Kaiser Permanente, NW, Portland, OR, ${ }^{b}$ Kaiser Permanente, NCa, San Jose, CA, USA

We describe an apparent balanced reciprocal translocation $(10 ; 12)$ (q26.1;q23.2) that was originally ascertained in a 3-year-old being evaluated for a developmental speech disorder. The child is otherwise nondysmorphic and a healthy appearing boy. The chromosome rearrangement was determined to be paternally derived in parental chromosome studies. In follow-up genetics clinic the father was soft spoken with a reported history of speech delay treated in grade school, and with an extended family history of some speech delay.
In spite of the translocation looking balanced with conventional G-banding and the familial nature of the rearrangement, both the father and son were evaluated with array based comparative genomic hybridization $(\mathrm{aCGH})$ using a $44 \mathrm{~K}$ oligonucleotide microarray (Agilent Technologies). The aCGH showed a $3 \mathrm{MB}$ deletion between $12 \mathrm{q} 21.31$-q21.33 in both the father and the son, with essentially all of $12 \mathrm{q} 21.32$ having apparently been deleted. The translocation breakpoints were subsequently revised to $t(10 ; 12)(\mathrm{q} 23.2 ; \mathrm{q} 21.32)$ to reflect the aCGH.

While aCGH based microdeletions and duplications have explained the more dramatic phenotypes seen with supposedly de novo balanced reciprocal translocations or more complex rearrangements $(\sim 40 \%$, De Gregori et. al., J Med Genet 2007;44:750-762), this case is exceptional in both its familial nature, and its relatively mild phenotype. The case also has implication with respect to our current management of familial rearrangements in prenatal testing and more broadly in clinical genetics.

\section{0 \\ Quality Watch: Detection and Reporting Program to Assist Quality Genetic Testing}

D.F.Saxe, ${ }^{a, b}$, J. Benkendorf ${ }^{b}$, T.M. Cowan ${ }^{b, c}$, T.W. Prior ${ }^{b, d}$, C.S. Richards ${ }^{b, e}$, K.W. Rao ${ }^{b, f}$, M. S. Watson ${ }^{b}$, and the ACM L Laboratory Quality Assurance Committee ${ }^{b}$

${ }^{a}$ Department of Pathology and Laboratory Medicine, Emory University School of Medicine, Atlanta, GA, bAmerican College of Medical Genetics, Bethesda, MD, 'Department of Pathology, Stanford University Medical Center, Stanford, CA, ${ }^{\mathrm{d}}$ Department of Pathology, Ohio State University, Columbus, $\mathrm{OH}$, ${ }^{e}$ Department of Molecular and Medical Genetics, Oregon Health Sciences University, Portland, OR, ${ }^{\mathrm{f}}$ Departments of Pediatrics and

Pathology, UNC, Chapel Hill, NC, USA

In 2005 the Laboratory Quality Assurance Committee (Lab QA) of the American College of Medical Genetics (ACMG) established the ACMG Quality Watch Subcommittee, (QWC), to address specific issues in clinical genetic testing related to product quality. The committee is now finalizing the program and hopes to be available in the next few months. The Quality Watch program functions through a link to the ACMG website which is designed to offer laboratory geneticists an avenue to identify possible defects in products used in clinical genetic testing is a rapid manner. The LIST SERV is accessible through the ACMG website, and will be available to ACMG and Association of Molecular Pathology (AMP) members only. The website is the initial communication venue for laboratory directors to discuss a putative problem, and assess if it has occurred often enough to merit further investigation. The program is moderated by a committee member from each laboratory specialty who acts as a facilitator in determining if a particular product is not working as directed. A moderator reviews all submissions, correlates them with similar incidents in other laboratories, and if determined to be a valid issue, contacts the appropriate laboratory specialty to investigate the submission. The LIST SERV is not an open forum, rather the link contains submission forms and directions on how to submit a problem and how other laboratories will be involved and receive information on the outcome of the investigation.

The details of how to submit a putative problem and participate in the process will be discussed. 